Prepared in cooperation with the U.S. Army Corps of Engineers-Fort Worth District, the Texas Water Development Board, the Guadalupe-Blanco River Authority, and the Edwards Aquifer Authority

\title{
A Preliminary Assessment of Streamflow Gains and Losses for Selected Stream Reaches in the Lower Guadalupe River Basin, Texas, 2010-12
}

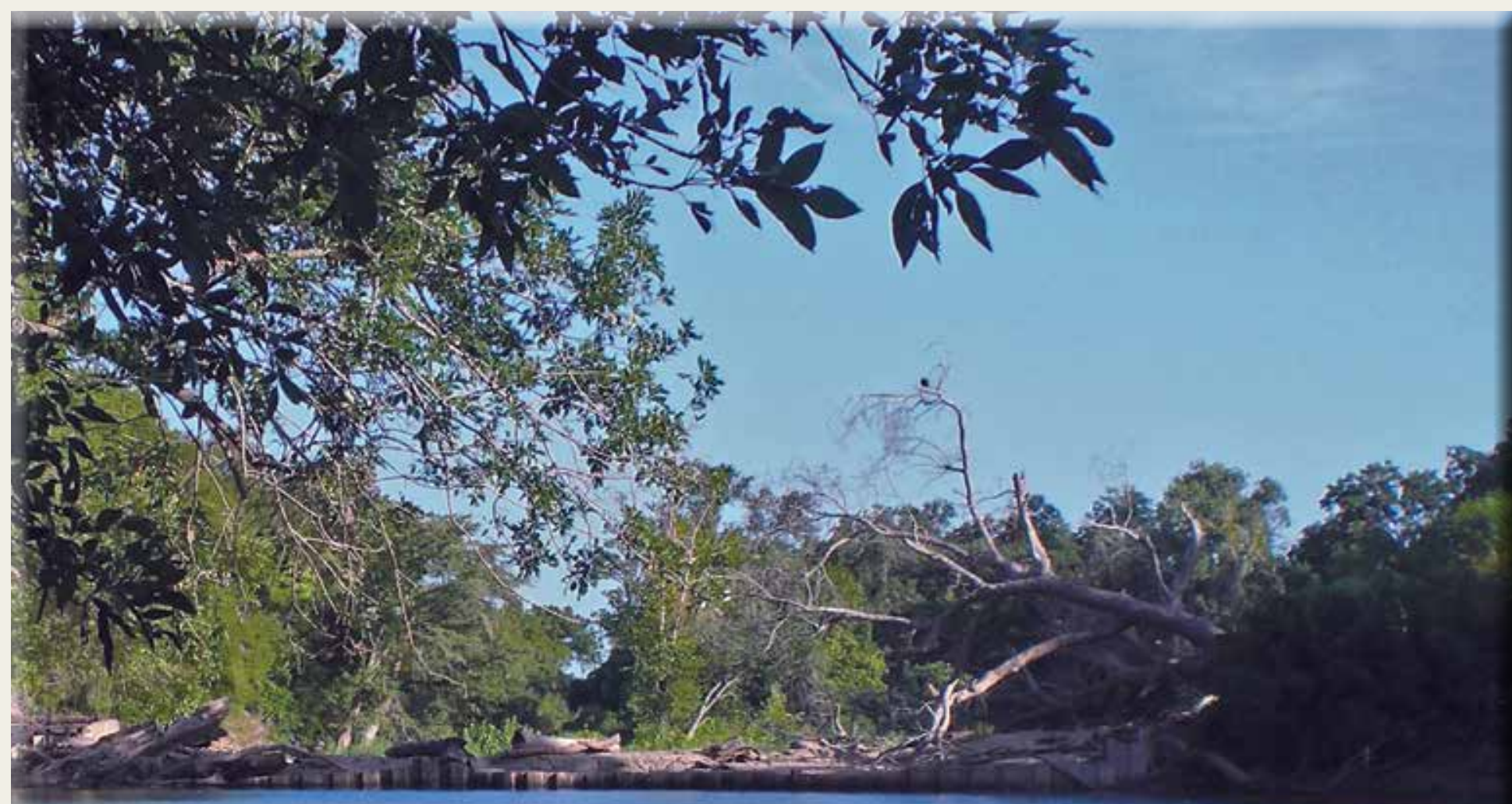

Scientific Investigations Report 2013-5209 
Cover, Photograph looking downstream from U.S. Geological Survey (USGS) streamflow-gaging station 08169840 Guadalupe River at Oak Forest, Texas, August 9, 2011. Photograph by Mark A. Warzecha, USGS. 


\section{A Preliminary Assessment of Streamflow Gains and Losses for Selected Stream Reaches in the Lower Guadalupe River Basin, Texas, 2010-12}

By Loren L. Wehmeyer, Karl E. Winters, and Darwin J. Ockerman

Prepared in cooperation with the U.S. Army Corps of Engineers-Fort Worth District, the Texas Water Development Board, the Guadalupe-Blanco River Authority, and the Edwards Aquifer Authority

Scientific Investigations Report 2013-5209 


\title{
U.S. Department of the Interior \\ SALLY JEWELL, Secretary
}

\author{
U.S. Geological Survey \\ Suzette M. Kimball, Acting Director
}

U.S. Geological Survey, Reston, Virginia: 2013

For more information on the USGS - the Federal source for science about the Earth, its natural and living resources, natural hazards, and the environment, visit http://www.usgs.gov or call 1-888-ASK-USGS.

For an overview of USGS information products, including maps, imagery, and publications, visit http://www.usgs.gov/pubprod

To order this and other USGS information products, visit http://store.usgs.gov

Any use of trade, firm, or product names is for descriptive purposes only and does not imply endorsement by the U.S. Government.

Although this information product, for the most part, is in the public domain, it also may contain copyrighted materials as noted in the text. Permission to reproduce copyrighted items must be secured from the copyright owner.

Suggested citation:

Wehmeyer, L.L., Winters, K.E., and Ockerman, D.J., 2013, A preliminary assessment of streamflow gains and losses for selected stream reaches in the lower Guadalupe River Basin, Texas, 2010-12: U.S. Geological Survey Scientific Investigations Report 2013-5209, 30 p., http://dx.doi.org/10.3133/20135209.

ISSN 2328-031X (print)

ISSN 2328-0328 (online) 


\section{Contents}

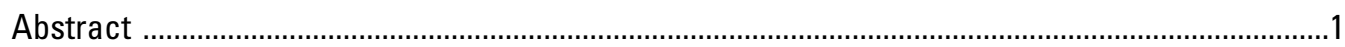

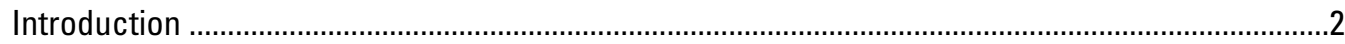

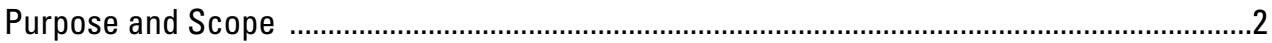

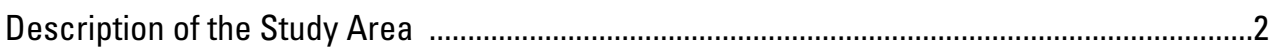

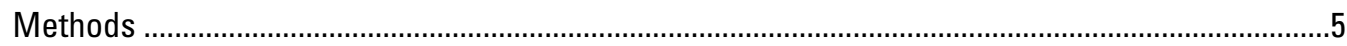

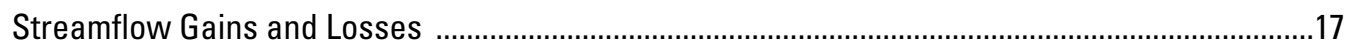

Streamflow Gains and Losses during March 15-21, $2010 \ldots \ldots \ldots \ldots \ldots \ldots \ldots \ldots \ldots \ldots \ldots \ldots \ldots \ldots \ldots \ldots \ldots \ldots . . .22$

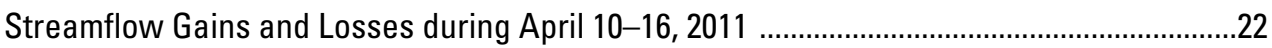

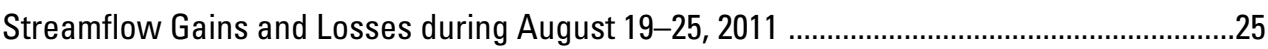

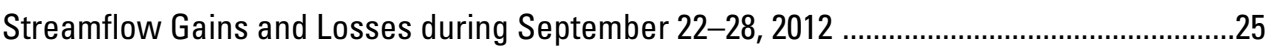

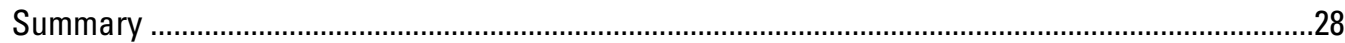

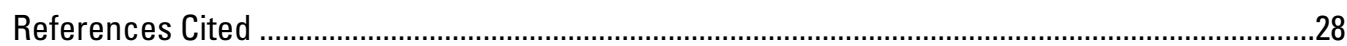

\section{Figures}

1. Map showing U.S. Geological Survey streamflow-gaging stations and stream reaches defined for assessment of streamflow gains and losses in the lower Guadalupe River Basin, south-central Texas

2. Map showing surficial geology and U.S. Geological Survey streamflow-gaging stations in the lower Guadalupe River Basin, south-central Texas

3. Map showing aquifer systems and U.S. Geological Survey streamflow-gaging stations in the lower Guadalupe River Basin, south-central Texas

4. Map showing location of inflow sites and U.S. Geological Survey streamflowgaging stations used in the lower Guadalupe River Basin gain and loss study

5. Map showing water right permit locations and U.S. Geological Survey streamflow-gaging stations, lower Guadalupe River Basin, south-central Texas .........14

6. Map showing streamflow gains and losses, March 15-21, 2010, lower Guadalupe River Basin, south-central Texas

7. Maps showing streamflow gains and losses, April 10-16, 2011, lower Guadalupe River Basin, south-central Texas

8. Maps showing streamflow gains and losses, August 19-25, 2011, lower Guadalupe River Basin, south-central Texas

9. Map showing streamflow gains and losses, September 22-28, 2012, in a reach of the Guadalupe River from Seguin, Texas, to Gonzales, Tex., lower Guadalupe River Basin, south-central Texas 


\section{Tables}

1. Selected U.S. Geological Survey streamflow-gaging stations in the lower Guadalupe River Basin, south-central Texas

2. Stream reaches for which streamflow conditions were analyzed in the lower Guadalupe River Basin, south-central Texas .8

3. Daily streamflow statistics for water years 1987-2011 for selected U.S. Geological Survey streamflow-gaging stations in the lower Guadalupe River Basin, south-central Texas

4. Inflow sites used for gain and loss computations in the lower Guadalupe River Basin, south-central Texas

5. Outflow sites used for gain and loss computations in the lower Guadalupe River Basin, south-central Texas

6. Base-flow periods in 2010 and 2011 selected for computing streamflow gains and losses in the lower Guadalupe River Basin, south-central Texas

7. Streamflow gains and losses computed for 21 stream reaches in the lower Guadalupe River Basin, south-central Texas, March 15-21, 2010

8. Streamflow gains and losses computed for 21 stream reaches in the lower Guadalupe River Basin, south-central Texas, April 10-16, 2011

9. Streamflow gains and losses computed for 21 stream reaches in the lower Guadalupe River Basin, south-central Texas, August 19-25, 2011

10. Streamflow gains and losses computed for a stream reach on the Guadalupe River from Seguin, Texas, to Gonzales, Tex., September 22-28, 2012 


\section{Conversion Factors}

Inch/Pound to SI

\begin{tabular}{lcl}
\hline \multicolumn{1}{c}{ Multiply } & By & \multicolumn{1}{c}{ To obtain } \\
\hline inch (in) & Length & centimeter $(\mathrm{cm})$ \\
inch (in) & 2.54 & millimeter $(\mathrm{mm})$ \\
foot (ft) & 25.4 & meter $(\mathrm{m})$ \\
mile (mi) & 0.3048 & kilometer $(\mathrm{km})$ \\
\hline & 1.609 & \\
\hline square mile $\left(\mathrm{mi}^{2}\right)$ & Area & hectare $(\mathrm{ha})$ \\
square mile $\left(\mathrm{mi}^{2}\right)$ & 259.0 & square kilometer $\left(\mathrm{km}^{2}\right)$ \\
\hline & 2.590 & cubic meter $\left(\mathrm{m}^{3}\right)$ \\
\hline acre-foot $(\mathrm{acre}-\mathrm{ft})$ & Volume & cubic hectometer $\left(\mathrm{hm}^{3}\right)$ \\
acre-foot $(\mathrm{acre}-\mathrm{ft})$ & 1,233 & \\
\hline & 0.001233 & cubic meter per second $\left(\mathrm{m}^{3} / \mathrm{s}\right)$ \\
\hline $\begin{array}{l}\text { cubic foot per second }\left(\mathrm{ft}^{3} / \mathrm{s}\right) \\
\text { cubic foot per second per square } \\
\text { mile }\left[\left(\mathrm{ft}^{3} / \mathrm{s}\right) / \mathrm{mi}^{2}\right]\end{array}$ & Flow rate & cubic meter per second per \\
\hline
\end{tabular}

Temperature in degrees Fahrenheit $\left({ }^{\circ} \mathrm{F}\right)$ may be converted to degrees Celsius $\left({ }^{\circ} \mathrm{C}\right)$ as follows:

${ }^{\circ} \mathrm{C}=\left({ }^{\circ} \mathrm{F}-32\right) / 1.8$

Vertical coordinate information is referenced to the North American Vertical Datum of 1988 (NAVD 88).

Horizontal coordinate information is referenced to the North American Datum of 1983 (NAD 83).

A water year is the 12-month period October 1 through September 30 designated by the calendar year in which it ends. 



\title{
A Preliminary Assessment of Streamflow Gains and Losses for Selected Stream Reaches in the Lower Guadalupe River Basin, Texas, 2010-12
}

\author{
By Loren L. Wehmeyer, Karl E. Winters, and Darwin J. Ockerman
}

\section{Abstract}

The U.S. Geological Survey, in cooperation with the U.S. Army Corps of Engineers-Fort Worth District, the Texas Water Development Board, the Guadalupe-Blanco River Authority, and the Edwards Aquifer Authority, investigated streamflow gains and losses in the lower Guadalupe River Basin during four selected base-flow periods in March 2010, April 2011, August 2011, and, for a stream reach between Seguin, Tex., and Gonzales, Tex., in September 2012. Major sources of streamflow in this basin include releases from Canyon Lake, inflow from major springs (Comal Springs, San Marcos Springs, and Hueco Springs), and base flow (groundwater seeping to streams). Streamflow and springflow data were collected at 35 streamflow-gaging stations (including 6 deployed for this study) during the base-flow periods. This report describes streamflow in the lower Guadalupe River Basin, which consists of the Guadalupe River drainage basin downstream from Canyon Lake to the Guadalupe River near Tivoli, Tex.

Streamflow conditions in the lower Guadalupe River Basin were analyzed by computing surface-water budgets for reaches of the lower Guadalupe River and tributary streams. Streamflow gains and losses were mapped for reaches where the computed gain or loss was greater than the uncertainty in the computed streamflow at the upstream and downstream ends of the reach.

During the March 15-21, 2010, base-flow period, five reaches had gains greater than the uncertainty in the computed streamflow, including reach 1 on the Guadalupe River, which gained 130 cubic feet per second $\left(\mathrm{ft}^{3} / \mathrm{s}\right)$, and reach 3 on the Comal River, which gained $359 \mathrm{ft}^{3} / \mathrm{s}$. Streamflow gains during March 2010 primarily were derived from (1) inflow from the Edwards aquifer outcrop, including Hueco Springs and Comal Springs; (2) flow conveyed through the alluvium of the streambed; (3) inflows from the Carrizo-Wilcox aquifer and the Yegua Jackson aquifer; and (4) groundwater inflows from the Gulf Coast aquifer, which are enhanced by seepage losses from Coleto Creek Reservoir. During this base-flow period, none of the reaches had a loss greater in magnitude than the uncertainty in the computed streamflow.
During the April 10-16, 2011, base-flow period, three reaches had gains greater than the uncertainty in the computed streamflow. Among these three reaches were reach 1 on the Guadalupe River, which gained $40.7 \mathrm{ft}^{3} / \mathrm{s}$, and reach 3 on the Comal River, which gained $271 \mathrm{ft}^{3} / \mathrm{s}$ - reaches where streamflow gains were also measured in March 2010. Streamflow gains during April 2011 primarily were derived from (1) inflow from the Edwards aquifer outcrop, including Hueco Springs and Comal Springs; and (2) inflows from the Carrizo-Wilcox aquifer. During this base-flow period, three reaches had losses greater in magnitude than the uncertainty in the computed streamflow. A reach of the Blanco River near Kyle, Tex. (reach 10), lost 18.7 cubic feet per second ( $\left.\mathrm{ft}^{3} / \mathrm{s}\right)$. Much of this loss likely entered the groundwater system through the numerous faults that intersect the stream channel northwest of Kyle. The reach that included the confluence of the Guadalupe and San Marcos Rivers (reach 17) lost $155 \mathrm{ft}^{3} / \mathrm{s}$, likely as recharge to the Sparta and Queen City aquifers.

During the August 19-25, 2011, base-flow period, three reaches had gains greater than the uncertainty in the computed streamflow, including reach 3 on the Comal River $\left(168 \mathrm{ft}^{3} / \mathrm{s}\right.$ gain), which was one of the reaches where gains in streamflow also were measured in March 2010 and April 2011. Streamflow gains in August 2011 were primarily from (1) inflows from Comal Springs, (2) inflows from the Yegua Jackson aquifer, and (3) groundwater inflows from the Gulf Coast aquifer, which are enhanced by seepage losses from Coleto Creek Reservoir. During this base-flow period, five reaches had losses greater in magnitude than the uncertainty in the computed streamflow. The reach including the confluence of the Guadalupe and Comal Rivers lost $82.8 \mathrm{ft}^{3} / \mathrm{s}$. Much of that loss likely seeped into the local groundwater system. The reach of the Guadalupe River south of New Braunfels, Tex., to Seguin, Tex., lost $53.5 \mathrm{ft}^{3} / \mathrm{s}$. Part of that loss may have been from seepage through streambed alluvium. Reaches 9 and 10 of the Blanco River near Kyle lost 2.20 and $6.60 \mathrm{ft}^{3} / \mathrm{s}$, respectively, likely as infiltration through numerous faults intersecting the stream channel northwest of Kyle. Plum Creek between Lockhart, Tex., and Luling, Tex., lost $2.11 \mathrm{ft}^{3} / \mathrm{s}$, likely as recharge to the Carrizo-Wilcox aquifer. A base-flow period during September 22-28, 2012, was studied for the reach of 
the Guadalupe River between Seguin and Gonzalez, including flows from San Marcos River and Plum Creek. During this period, for the Guadalupe River reach between Seguin and Oak Forest, no computed gains or losses were greater in magnitude than the uncertainty in the computed streamflow.

\section{Introduction}

In south-central Texas, the lower Guadalupe River and its tributaries provide water for municipal water supplies, farms, ranches, industries, recreational activities, wildlife, and wastewater assimilation. The Guadalupe River Basin includes multiple springs that help sustain streamflow in some stream reaches and provides habitat for several endangered and threatened species (Ockerman and Slattery, 2008).

Streamflow conditions in the lower Guadalupe River Basin are affected by rainfall-runoff processes, outflows (withdrawals) for water supplies, point-source inflows, reservoir operations, spring flows, and infiltration. During normal base-flow conditions, releases from Canyon Lake and inflows from major springs (Comal, San Marcos, and Hueco Springs) (fig. 1) account for most of the streamflow in the lower Guadalupe River. A better understanding of streamflow conditions in the basin, including how gains, losses, outflows, and inflows affect downstream flows, can help resource managers to design watershed-management and operation strategies that improve utilization of available water resources in this basin.

In a previous study, the U.S. Geological Survey (USGS), in cooperation with the Edwards Aquifer Authority (EAA), evaluated streamflow conditions in the Guadalupe River Basin for the period 1987-2006 and described streamflow gains and losses and relative contributions of major springs to streamflow (Ockerman and Slattery, 2008). That report used historical streamflow data and available outflow and inflow data to evaluate streamflow characteristics of reaches in the Guadalupe River Basin and to estimate the contributions of major springs to streamflow in the lower part of the basin (downstream from Canyon Lake) for long-term (20-year average) conditions and selected short-term base-flow periods.

\section{Purpose and Scope}

The purpose of this report is to provide a preliminary assessment of streamflow gains and losses in the lower Guadalupe River Basin downstream from Canyon Lake. Streamflow gains and losses for certain stream reaches were evaluated for four selected periods of base flow during 2010-12-March 2010, April 2011, August 2011, and September 2012. The assessment of streamflow in September 2012 was limited to the Guadalupe River between Seguin, Tex., and Gonzales, Tex., and the San Marcos River between Luling, Tex., and Gonzales, Tex. Streamflow and spring-flow data were collected at 35 streamflow-gaging stations in the study area (fig. 1; table 1), including 6 deployed for this study, during the selected base-flow periods from 2010-11, and at 2 partial-record stations in September 2012.

The study results presented in this report do not constitute a comprehensive assessment of streamflow gains and losses in the lower Guadalupe River Basin because many factors were not incorporated in the assessment; for example, the effects of hydropower generation on streamflow, gains or losses to bank storage, interaction of surface water and groundwater, underflow in the streambed alluvium, and evapotranspiration losses are addressed only in part. Also, the extent of possible unpermitted withdrawals is unknown and therefore not included in the assessment.

\section{Description of the Study Area}

The headwaters of the Guadalupe River are in southwestern Kerr County, Tex. From there, the river flows easterly to southeasterly for about 250 miles (mi) to Gonzalez, Tex., then southeasterly for another $150 \mathrm{mi}$ to join the San Antonio River about $11 \mathrm{mi}$ upstream from the San Antonio Bay on the Gulf of Mexico (fig. 1). The study area for this report is the lower Guadalupe River Basin, which includes the basin downstream from Canyon Lake to the Guadalupe River near Tivoli, Tex. The entire Guadalupe River Basin includes about 10,100 square miles $\left(\mathrm{mi}^{2}\right)$. The lower Guadalupe River Basin study area includes approximately 8,690 $\mathrm{mi}^{2}$.

The Blanco River, San Marcos River, and San Antonio River are principal tributaries of the Guadalupe River. The two major reservoirs in the Guadalupe River Basin are Canyon Lake and Coleto Creek Reservoir. Canyon Lake impounds the Guadalupe River in Comal County, Tex., about $12 \mathrm{mi}$ northwest of New Braunfels, Tex. Canyon Lake impounds runoff from $1,432 \mathrm{mi}^{2}$ of drainage area and has 382,000 acrefeet (acre-ft) of authorized conservation storage (GuadalupeBlanco River Authority, 2007a). Construction of the dam and reservoir at Canyon Lake began in 1958 and impoundment began in 1964. Coleto Creek Reservoir impounds Coleto Creek and Perdido Creek, about 12 mi southwest of Victoria, Tex. The dam for that reservoir was completed in 1980 and impounds runoff from $507 \mathrm{mi}^{2}$ of drainage area. Conservation storage for that reservoir is 35,060 acre-ft (Guadalupe-Blanco River Authority, 2007b). The primary purpose of that reservoir is to provide cooling water for electric power generation. Daily regulation of streamflow for power generation affects much of the Guadalupe River downstream from Canyon Lake.

Major population centers in the Guadalupe River Basin include Kerrville, New Braunfels, San Marcos, Seguin, Lockhart, Gonzales, Cuero, Luling, and Victoria, Tex. The 2009 population of the basin was approximately 646,000 based on the 2009 estimated population of Kerr, Kendall, Comal, Hays, Guadalupe, Caldwell, Gonzales, De Witt, Goliad, and Victoria Counties (U.S. Census Bureau, 2011). Agriculture is the primary land use in the study area (MultiResolution Land Characteristics Consortium, 2006). 


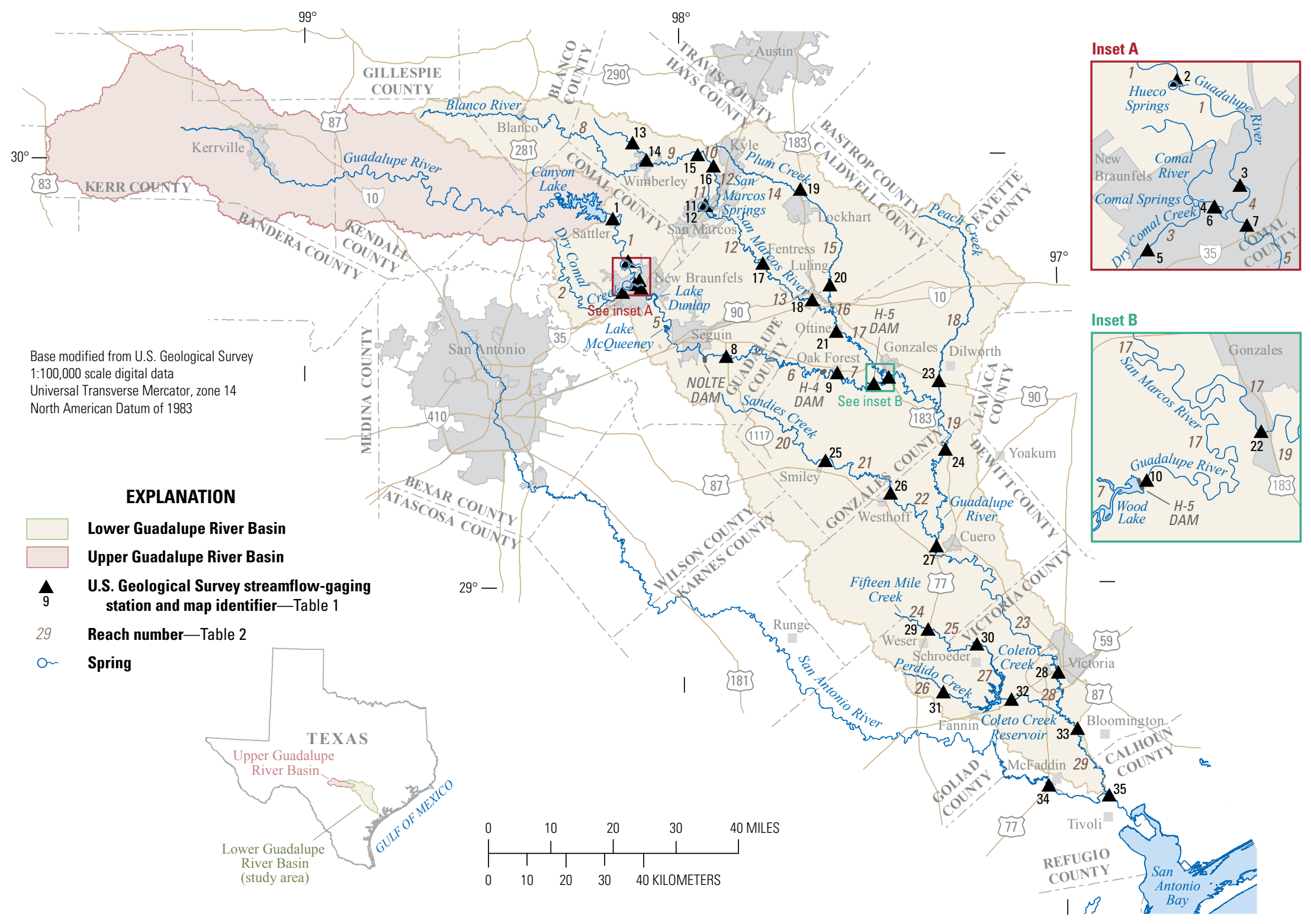

Figure 1. U.S. Geological Survey streamflow-gaging stations and stream reaches defined for assessment of streamflow gains and losses in the lower Guadalupe River Basin, south-central Texas. 
Table 1. Selected U.S. Geological Survey streamflow-gaging stations in the lower Guadalupe River Basin, south-central Texas.

[--, not applicable; FM, Farm Road; a water year is the 12-month period from October 1 through September 30 designated by the calendar year in which it ends]

\begin{tabular}{|c|c|c|c|c|c|c|c|}
\hline $\begin{array}{l}\text { Map } \\
\text { identifier } \\
\text { (fig. 1) }\end{array}$ & $\begin{array}{c}\text { Streamflow- } \\
\text { gaging } \\
\text { station } \\
\text { number }\end{array}$ & Station name & County & $\begin{array}{l}\text { Period of record } \\
\text { (water years) }\end{array}$ & $\begin{array}{c}\text { Latitude } \\
\text { (degrees, } \\
\text { minutes, } \\
\text { seconds) }\end{array}$ & $\begin{array}{l}\text { Longitude } \\
\text { (degrees, } \\
\text { minutes, } \\
\text { seconds) }\end{array}$ & $\begin{array}{c}\text { Drainage } \\
\text { area } \\
\text { (square } \\
\text { miles) }\end{array}$ \\
\hline 1 & 08167800 & Guadalupe River at Sattler, Tex. & Comal & 1960-present & $29^{\circ} 51^{\prime} 32^{\prime \prime}$ & $98^{\circ} 10^{\prime} 47^{\prime \prime}$ & 1,436 \\
\hline 2 & 08168000 & Hueco Springs near New Braunfels, Tex. & Comal & 2003-present & $29^{\circ} 45^{\prime} 33^{\prime \prime}$ & $98^{\circ} 08^{\prime} 23^{\prime \prime}$ & -- \\
\hline 3 & 08168500 & Guadalupe River above Comal River at New Braunfels, Tex. & Comal & 1928-present & $29^{\circ} 42^{\prime} 53^{\prime \prime}$ & $98^{\circ} 06^{\prime} 35^{\prime \prime}$ & 1,518 \\
\hline 4 & 08168710 & Comal Springs at New Braunfels, Tex. & Comal & 1928-present & $29^{\circ} 42^{\prime} 21^{\prime \prime}$ & $98^{\circ} 07^{\prime} 20^{\prime \prime}$ & -- \\
\hline 5 & 08168797 & Dry Comal Creek at Loop 337 near New Braunfels, Tex. & Comal & 2006-present & $29^{\circ} 41^{\prime} 16.8^{\prime \prime}$ & $98^{\circ} 09^{\prime} 17.4^{\prime \prime}$ & 107 \\
\hline 6 & 08169000 & Comal River at New Braunfels, Tex. & Comal & 1928-present & $29^{\circ} 42^{\prime} 21^{\prime \prime}$ & $98^{\circ} 07^{\prime} 20^{\prime \prime}$ & 130 \\
\hline 7 & 08169500 & Guadalupe River at New Braunfels, Tex. & Comal & 1915-98 & $29^{\circ} 41^{\prime} 52^{\prime \prime}$ & $98^{\circ} 06^{\prime} 23^{\prime \prime}$ & 1,652 \\
\hline 8 & 08169792 & Guadalupe River at FM 1117 near Seguin, Tex. & Guadalupe & 2005-present & $29^{\circ} 32^{\prime} 10.2^{\prime \prime}$ & $97^{\circ} 52^{\prime} 51.4^{\prime \prime}$ & 1,957 \\
\hline 9 & 08169840 & Guadalupe River at Oak Forest, Tex. & Gonzales & $2010-12^{\mathrm{a}}$ & $29^{\circ} 29^{\prime} 44.31^{\prime \prime}$ & $97^{\circ} 35^{\prime} 12.56^{\prime \prime}$ & 2,068 \\
\hline 10 & 08169860 & Guadalupe River below H-5 Dam near Gonzales, Tex. & Gonzales & $2010-12^{\mathrm{a}}$ & $29^{\circ} 28^{\prime} 12.11^{\prime \prime}$ & $97^{\circ} 29^{\prime} 24.61^{\prime \prime}$ & 2,099 \\
\hline 11 & 08170000 & San Marcos Springs at San Marcos, Tex. & Hays & 1956-present & $29^{\circ} 53^{\prime} 20^{\prime \prime}$ & $97^{\circ} 56^{\prime} 02^{\prime \prime}$ & -- \\
\hline 12 & 08170500 & San Marcos River at San Marcos, Tex. & Hays & 1915-present & $29^{\circ} 53^{\prime} 20^{\prime \prime}$ & $97^{\circ} 56^{\prime} 02^{\prime \prime}$ & 49 \\
\hline 13 & 08170990 & Jacobs Well Spring near Wimberley, Tex. & Hays & 2005-present & $30^{\circ} 02^{\prime} 04^{\prime \prime}$ & $98^{\circ} 07^{\prime} 34^{\prime \prime}$ & -- \\
\hline 14 & 08171000 & Blanco River at Wimberley, Tex. & Hays & 1924-present & $29^{\circ} 59^{\prime} 39^{\prime \prime}$ & $98^{\circ} 05^{\prime} 19^{\prime \prime}$ & 355 \\
\hline 15 & 08171290 & Blanco River at Halifax Ranch near Kyle, Tex. & Hays & 2009-present & $30^{\circ} 00^{\prime} 20^{\prime \prime}$ & $97^{\circ} 57^{\prime} 09^{\prime \prime}$ & 391 \\
\hline 16 & 08171300 & Blanco River near Kyle, Tex. & Hays & 1956-present & $29^{\circ} 58^{\prime} 45^{\prime \prime}$ & $97^{\circ} 54^{\prime} 35^{\prime \prime}$ & 412 \\
\hline 17 & 08171500 & San Marcos River at FM 20 at Fentress, Tex. & Caldwell & $2010-11^{\mathrm{a}}$ & $29^{\circ} 45^{\prime} 10.02^{\prime \prime}$ & $97^{\circ} 46^{\prime} 51.39^{\prime \prime}$ & 598 \\
\hline 18 & 08172000 & San Marcos River at Luling, Tex. & Caldwell & 1939-present & $29^{\circ} 39^{\prime} 58^{\prime \prime}$ & $97^{\circ} 39^{\prime} 02^{\prime \prime}$ & 838 \\
\hline 19 & 08172400 & Plum Creek at Lockhart, Tex. & Caldwell & 1959-present & $29^{\circ} 55^{\prime} 22^{\prime \prime}$ & $97^{\circ} 40^{\prime} 44^{\prime \prime}$ & 112 \\
\hline 20 & 08173000 & Plum Creek near Luling, Tex. & Caldwell & 1930-present & $29^{\circ} 41^{\prime} 58^{\prime \prime}$ & $97^{\circ} 36^{\prime} 12^{\prime \prime}$ & 309 \\
\hline 21 & 08173500 & San Marcos River at Ottine, Tex. & Gonzales & $1915-43,2010-11^{\mathrm{a}}$ & $29^{\circ} 35^{\prime} 33.39^{\prime \prime}$ & $97^{\circ} 35^{\prime} 17.12^{\prime \prime}$ & 1,249 \\
\hline 22 & 08173900 & Guadalupe River at Gonzales, Tex. & Gonzales & 1997-present & $29^{\circ} 29^{\prime} 03^{\prime \prime}$ & $97^{\circ} 27^{\prime} 00^{\prime \prime}$ & 3,490 \\
\hline 23 & 08174600 & Peach Creek below Dilworth, Tex. & Gonzales & 1959-present & $29^{\circ} 28^{\prime} 26^{\prime \prime}$ & $97^{\circ} 18^{\prime} 59^{\prime \prime}$ & 460 \\
\hline 24 & 08174700 & Guadalupe River at U.S. Highway 183 near Yoakum, Tex. & DeWitt & $2010-11^{\mathrm{a}}$ & $29^{\circ} 18^{\prime} 52.08^{\prime \prime}$ & $97^{\circ} 18^{\prime} 12.60^{\prime \prime}$ & 4,071 \\
\hline 25 & 08174970 & Sandies Creek near Smiley, Tex. & Gonzales & $2010-11^{\mathrm{a}}$ & $29^{\circ} 17^{\prime} 30.32^{\prime \prime}$ & $97^{\circ} 37^{\prime} 14.72^{\prime \prime}$ & 197 \\
\hline 26 & 08175000 & Sandies Creek near Westhoff, Tex. & DeWitt & 1930-present & $29^{\circ} 12^{\prime} 54^{\prime \prime}$ & $97^{\circ} 26^{\prime} 57^{\prime \prime}$ & 549 \\
\hline 27 & 08175800 & Guadalupe River at Cuero, Tex. & DeWitt & 1964-present & $29^{\circ} 05^{\prime} 25^{\prime \prime}$ & $97^{\circ} 19^{\prime} 46^{\prime \prime}$ & 4,934 \\
\hline 28 & 08176500 & Guadalupe River at Victoria, Tex. & Victoria & 1935-present & $28^{\circ} 47^{\prime} 34^{\prime \prime}$ & $97^{\circ} 00^{\prime} 46^{\prime \prime}$ & 5,198 \\
\hline 29 & 08176550 & Fifteenmile Creek near Weser, Tex. & De Witt & $1985-89$ & $28^{\circ} 53^{\prime} 51^{\prime \prime}$ & $97^{\circ} 21^{\prime} 17^{\prime \prime}$ & 167 \\
\hline 30 & 08176900 & Coleto Creek at Arnold Road Crossing near Schroeder, Tex. & Goliad & 1979-present & $28^{\circ} 51^{\prime} 41^{\prime \prime}$ & $97^{\circ} 13^{\prime} 34^{\prime \prime}$ & 357 \\
\hline 31 & 08177300 & Perdido Creek at FM 622 near Fannin, Tex. & Goliad & 1978-present & $28^{\circ} 45^{\prime} 05^{\prime \prime}$ & $97^{\circ} 19^{\prime} 01^{\prime \prime}$ & 28 \\
\hline 32 & 08177500 & Coleto Creek near Victoria, Tex. & Victoria & 1939-present & $28^{\circ} 43^{\prime} 51^{\prime \prime}$ & $97^{\circ} 08^{\prime} 18^{\prime \prime}$ & 500 \\
\hline 33 & 08177520 & Guadalupe River near Bloomington, Tex. & Victoria & 1999-present & $28^{\circ} 39^{\prime} 43^{\prime \prime}$ & $96^{\circ} 57^{\prime} 55^{\prime \prime}$ & 5,816 \\
\hline 34 & 08188570 & San Antonio River near McFaddin, Tex. & Refugio & 2006-present & $28^{\circ} 31^{\prime} 52.5^{\prime \prime}$ & $97^{\circ} 02^{\prime} 33.7^{\prime \prime}$ & 4,134 \\
\hline 35 & 08188800 & Guadalupe River near Tivoli, Tex. & Refugio & 2000-present & $28^{\circ} 30^{\prime} 20^{\prime \prime}$ & $96^{\circ} 53^{\prime} 04^{\prime \prime}$ & 10,128 \\
\hline
\end{tabular}

aPartial-record site deployed for this investigation. 
Three major springs are in the Guadalupe River Basin: Comal Springs, San Marcos Springs, and Hueco Springs (fig. 1). Comal Springs is the largest spring in the Southwest United States (Brune, 1975). Comal Springs discharges from several outlets and provides most of the flow in the Comal River, which joins the Guadalupe River at New Braunfels. The annual average (water years 1933-2010) discharge of Comal Springs was 291 cubic feet per second $\left(\mathrm{ft}^{3} / \mathrm{s}\right)$ (U.S. Geological Survey, 2012a). San Marcos Springs, also with several outlets, provides most of the base flow for the San Marcos River, which joins the Guadalupe River near Gonzales. The San Marcos Springs, collectively, are the second largest spring in Texas (Brune, 1975). The annual average discharge (water years 1957-2010) for San Marcos Springs was $175 \mathrm{ft}^{3} / \mathrm{s}$ (U.S. Geological Survey, 2012a). Hueco Springs is on the west side of the Guadalupe River about 3 mi upstream from New Braunfels. The annual average discharge (water years 2004-8) for Hueco Springs was $51.8 \mathrm{ft}^{3} / \mathrm{s}$ (U.S. Geological Survey, 2012a).

The surficial geology of the lower Guadalupe River Basin ranges in age from the Lower Cretaceous to the Quaternary period (fig. 2). Aquifer outcrops include the Gulf Coast, Yegua Jackson, Sparta, Queen City, Carrizo-Wilcox, Edwards, Edwards-Trinity, and Trinity aquifers (fig. 3). These strata dip to the southeast and contain various interstitial chalk and clay layers (fig. 2).

Numerous faults are present in the chalk and limestone formations in the upper part of the study area (fig. 2) (Hanson and Small, 1995; U.S. Geological Survey, 2012b). Many of these faults intersect the channels of the streams crossing the outcrops of the Edwards aquifer. Most losses observed in streams crossing the Edwards aquifer are the result of streamflow contributing to groundwater recharge through faults intersecting the channels (Slade and others, 1986; Pantea and Cole, 2004).

The climate of the study area is subtropical, subhumid and is characterized by hot summers and mild winters (Larkin and Bomar, 1983). Most rainfall in the area occurs in spring, early summer, and fall. Periods with relatively large or small amounts of rainfall are common, resulting in recurring floods and droughts. Average annual rainfall (1971-2000) at the National Weather Service station at New Braunfels was 35.74 inches per year (National Oceanic and Atmospheric Administration, 2002). In the region, water-balance modeling indicates that more than 80 percent of rainfall might be evaporated and transpired (Lizárraga and Ockerman, 2010). Rainfall greater than 0.01 inch was measured, on average, for 77 days per year during 1971-2000. Average monthly low temperatures from 1971-2000 ranged from 35.5 degrees Fahrenheit $\left({ }^{\circ} \mathrm{F}\right)$ in January to $70.6{ }^{\circ} \mathrm{F}$ in July. Average monthly high temperatures from 1971-2000 ranged from $61.7^{\circ} \mathrm{F}$ in January to $95.3^{\circ} \mathrm{F}$ in August (National Oceanic and Atmospheric Administration, 2012).

\section{Methods}

To help evaluate streamflow gains and losses, streamflow conditions in the lower Guadalupe River Basin were analyzed by computing surface-water budgets for reaches of the lower Guadalupe River and tributary streams during 1987-2011. The lower Guadalupe River Basin was divided into a network of 29 stream reaches (table 2), defined by locations of 31 of the 35 USGS streamflow-gaging stations shown in figure 1. Of the 31 streamflow-gaging stations used to define the reaches, 6 were partial-record stations established to provide streamflow data for the selected base-flow periods used for analysis in this report and thus do not have long-term data from which to compute streamflow statistics. Some stream reaches include more than one upstream streamflow-gaging station because they include the confluence of streams.

Daily streamflow statistics were computed for 17 USGS streamflow-gaging stations in the lower Guadalupe River Basin with at least 10 years of record during the 25-year period from 1987-2011 (table 3). Those statistics include daily mean streamflow, 20 percent exceedance streamflow, 50 percent exceedance (median) streamflow, 80 percent exceedance streamflow, and 90 percent exceedance streamflow. The percentage exceedance streamflow is defined as the daily mean streamflow that was exceeded for the specified percentage of time during a base-flow period. For example, the 90-percent exceedance streamflow represents a (relatively low) daily mean streamflow that was exceeded during 90 percent of the base-flow period. The period 19872011 provides a long-term period of record for comparison among many streamflow-gaging stations in the lower Guadalupe River Basin.

Daily streamflow data from USGS streamflow-gaging stations used for analysis were obtained from the USGS National Water Information System (NWISWeb) (U.S. Geological Survey, 2012a). These data were collected by the USGS in cooperation with Federal, State, and local agencies, including the U.S. Army Corps of Engineers-Fort Worth District, the Texas Water Development Board (TWDB), the Guadalupe-Blanco River Authority, and the Edwards Aquifer Authority. Accuracy of the streamflow records vary in time and by streamflow-gaging station. The accuracy of streamflow records is considered "good," excluding estimated values, if 95 percent of the daily streamflows are within 10 percent of their true values (U.S. Geological Survey, 2012c). 


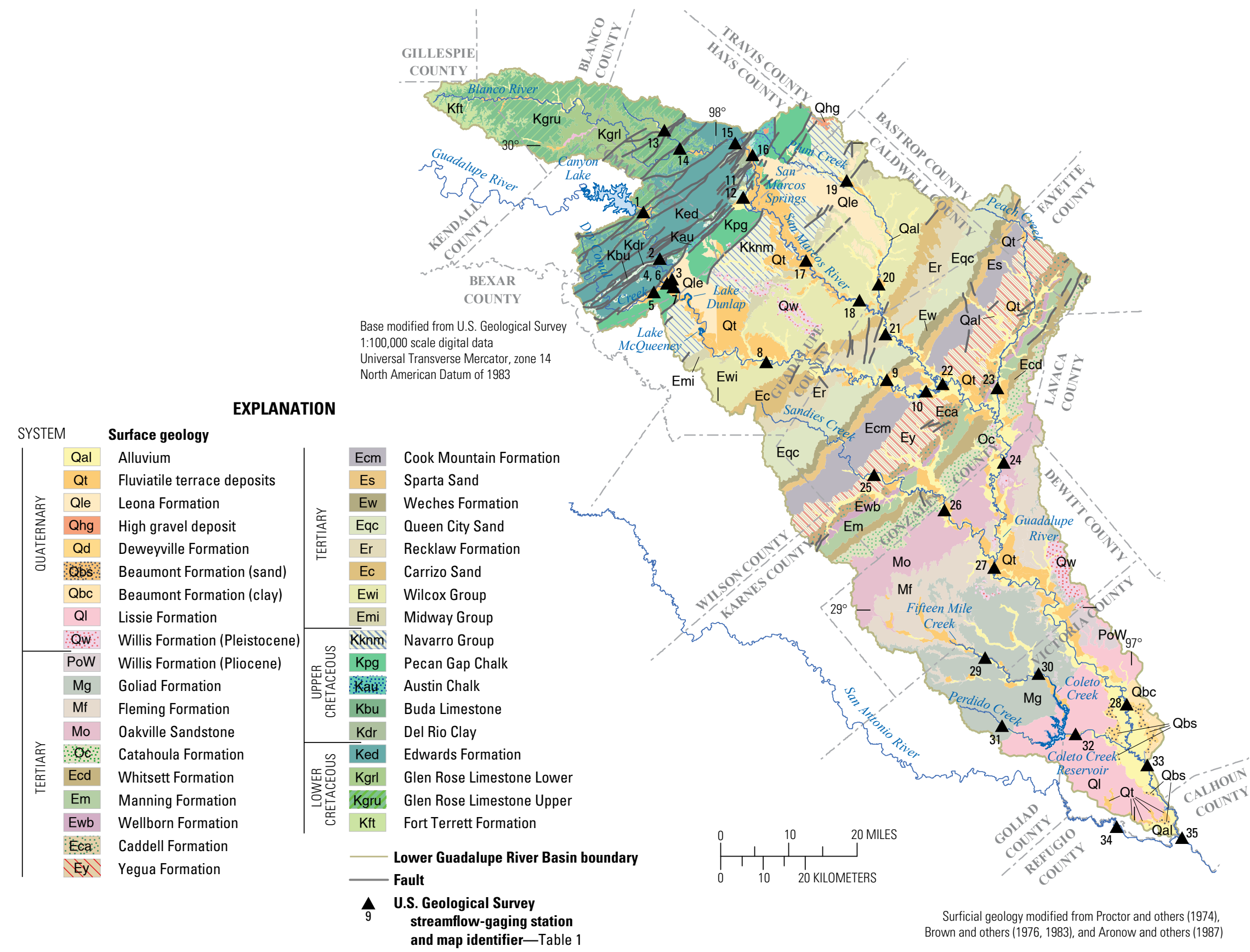

Figure 2. Surficial geology and U.S. Geological Survey streamflow-gaging stations in the lower Guadalupe River Basin, south-central Texas. 


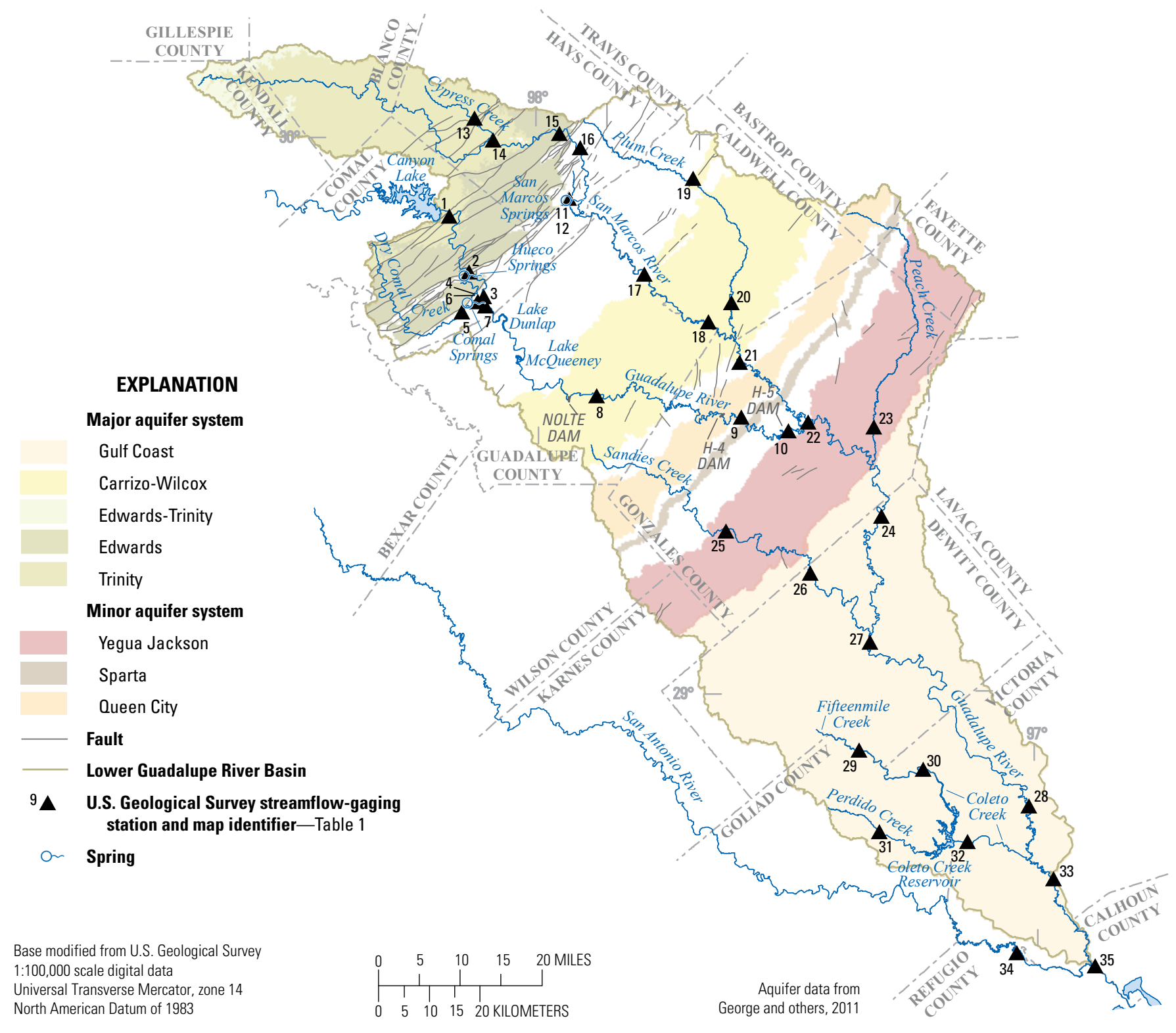

Figure 3. Aquifer systems and U.S. Geological Survey streamflow-gaging stations in the lower Guadalupe River Basin, south-central Texas. 
Table 2. Stream reaches for which streamflow conditions were analyzed in the lower Guadalupe River Basin, south-central Texas.

[--, not applicable because there is no upstream streamflow-gaging station; FM, Farm Road]

\begin{tabular}{|c|c|c|c|c|c|c|c|c|}
\hline \multirow[b]{2}{*}{$\begin{array}{c}\text { Reach } \\
\text { number } \\
\text { (figs. 1, } \\
\text { 7-9) }\end{array}$} & \multicolumn{3}{|c|}{ Upstream streamflow-gaging stations } & \multirow[b]{2}{*}{$\begin{array}{l}\text { Stream } \\
\text { reach } \\
\text { length } \\
\text { (miles) }\end{array}$} & \multicolumn{3}{|c|}{ Downstream streamflow-gaging stations } & \multirow[b]{2}{*}{$\begin{array}{c}\text { Cumulative } \\
\text { drainage area } \\
\text { at reach outlet } \\
\text { (square miles) }\end{array}$} \\
\hline & $\begin{array}{c}\text { Map } \\
\text { identi- } \\
\text { fier } \\
\text { (fig. 1) }\end{array}$ & $\begin{array}{l}\text { Station } \\
\text { number }\end{array}$ & Station name & & $\begin{array}{c}\text { Map } \\
\text { identi- } \\
\text { fier } \\
\text { (fig. 1) }\end{array}$ & $\begin{array}{l}\text { Station } \\
\text { number }\end{array}$ & Station name & \\
\hline 1 & 1 & 08167800 & Guadalupe River at Sattler, Tex. & 15 & 3 & 08168500 & $\begin{array}{l}\text { Guadalupe River above Comal River at New } \\
\text { Braunfels, Tex. }\end{array}$ & 1,518 \\
\hline 2 & -- & -- & -- & 29 & 5 & 08168797 & $\begin{array}{l}\text { Dry Comal Creek at Loop } 337 \text { near New } \\
\text { Braunfels, Tex. }\end{array}$ & 107 \\
\hline 3 & 5 & 08168797 & $\begin{array}{l}\text { Dry Comal Creek at Loop } 337 \text { near New } \\
\text { Braunfels, Tex. }\end{array}$ & 3.0 & 6 & 08169000 & Comal River at New Braunfels, Tex. & 130 \\
\hline \multirow[t]{2}{*}{4} & 3 & 08168500 & $\begin{array}{l}\text { Guadalupe River above Comal River at New } \\
\text { Braunfels, Tex. }\end{array}$ & 1.9 & 7 & 08169500 & Guadalupe River at New Braunfels, Tex. & 1,652 \\
\hline & 6 & 08169000 & Comal River at New Braunfels, Tex. & 1.8 & & & & \\
\hline 5 & 7 & 08169500 & Guadalupe River at New Braunfels, Tex. & 33 & 8 & 08169792 & Guadalupe River at FM 1117 near Seguin, Tex. & 1,957 \\
\hline 6 & 8 & 08169792 & Guadalupe River at FM 1117 near Seguin, Tex. & 43 & 9 & 08169840 & Guadalupe River at Oak Forest, Tex. & 2,068 \\
\hline 7 & 9 & 08169840 & Guadalupe River at Oak Forest, Tex. & 14 & 10 & 08169860 & $\begin{array}{l}\text { Guadalupe River below H-5 Dam near Gon- } \\
\text { zales, Tex. }\end{array}$ & 2,099 \\
\hline 8 & -- & -- & -- & 60 & 14 & 08171000 & Blanco River at Wimberley, Tex. & 355 \\
\hline 9 & 14 & 08171000 & Blanco River at Wimberley, Tex. & 12 & 15 & 08171290 & Blanco River at Halifax Ranch near Kyle, Tex. & 391 \\
\hline 10 & 15 & 08171290 & Blanco River at Halifax Ranch near Kyle, Tex. & 5.1 & 16 & 08171300 & Blanco River near Kyle, Tex. & 412 \\
\hline 11 & -- & -- & -- & 4.4 & 12 & 08170500 & San Marcos River at San Marcos, Tex. & 49 \\
\hline \multirow[t]{2}{*}{12} & 12 & 08170500 & San Marcos River at San Marcos, Tex. & 24 & 17 & 08171500 & San Marcos River at FM 20 at Fentress, Tex. & 598 \\
\hline & 16 & 08171300 & Blanco River near Kyle, Tex. & 33 & & & & \\
\hline 13 & 17 & 08171500 & San Marcos River at FM 20 at Fentress, Tex. & 42 & 18 & 08172000 & San Marcos River at Luling, Tex. & 838 \\
\hline 14 & -- & -- & -- & 22 & 19 & 08172400 & Plum Creek at Lockhart, Tex. & 112 \\
\hline 15 & 19 & 08172400 & Plum Creek at Lockhart, Tex. & 23 & 20 & 08173000 & Plum Creek near Luling, Tex. & 309 \\
\hline \multirow[t]{2}{*}{16} & 18 & 08172000 & San Marcos River at Luling, Tex. & 14 & 21 & 08173500 & San Marcos River at Ottine, Tex. & 1,249 \\
\hline & 20 & 08173000 & Plum Creek near Luling, Tex. & 12 & & & & \\
\hline
\end{tabular}


Table 2. Stream reaches for which streamflow conditions were analyzed in the lower Guadalupe River Basin, south-central Texas.-Continued

[--, not applicable because there is no upstream streamflow-gaging station; FM, Farm Road]

\begin{tabular}{|c|c|c|c|c|c|c|c|c|}
\hline \multirow{2}{*}{$\begin{array}{l}\text { Reach } \\
\text { number } \\
\text { (figs. 1, } \\
\text { 7-9) }\end{array}$} & \multicolumn{3}{|c|}{ Upstream streamflow-gaging stations } & \multirow[b]{2}{*}{$\begin{array}{l}\text { Stream } \\
\text { reach } \\
\text { length } \\
\text { (miles) }\end{array}$} & \multicolumn{3}{|c|}{ Downstream streamflow-gaging stations } & \multirow[b]{2}{*}{$\begin{array}{c}\text { Cumulative } \\
\text { drainage area } \\
\text { at reach outlet } \\
\text { (square miles) }\end{array}$} \\
\hline & $\begin{array}{l}\text { Map } \\
\text { identi- } \\
\text { fier } \\
\text { (fig. 1) }\end{array}$ & $\begin{array}{l}\text { Station } \\
\text { number }\end{array}$ & Station name & & $\begin{array}{l}\text { Map } \\
\text { identi- } \\
\text { fier } \\
\text { (fig. 1) }\end{array}$ & $\begin{array}{l}\text { Station } \\
\text { number }\end{array}$ & Station name & \\
\hline \multirow[t]{2}{*}{17} & 10 & 08169860 & $\begin{array}{l}\text { Guadalupe River below H-5 Dam near Gonza- } \\
\text { les, Tex. }\end{array}$ & 12 & 22 & 08173900 & Guadalupe River at Gonzales, Tex. & 3,490 \\
\hline & 21 & 08173500 & San Marcos River at Ottine, Tex. & 25 & & & & \\
\hline 18 & -- & -- & -- & 46 & 23 & 08174600 & Peach Creek below Dilworth, Tex. & 460 \\
\hline 19 & $\begin{array}{l}22 \\
23\end{array}$ & $\begin{array}{l}08173900 \\
08174600\end{array}$ & $\begin{array}{l}\text { Guadalupe River at Gonzales, Tex. } \\
\text { Peach Creek below Dilworth, Tex. }\end{array}$ & $\begin{array}{l}36 \\
19\end{array}$ & 24 & 08174700 & $\begin{array}{l}\text { Guadalupe River at U.S. Highway } 183 \text { near } \\
\text { Yoakum, Tex. }\end{array}$ & 4,071 \\
\hline 20 & -- & -- & -- & 56 & 25 & 08174970 & Sandies Creek near Smiley, Tex. & 197 \\
\hline 21 & 25 & 08174970 & Sandies Creek near Smiley, Tex. & 24 & 26 & 08175000 & Sandies Creek near Westhoff, Tex. & 549 \\
\hline \multirow[t]{2}{*}{22} & 24 & 08174700 & $\begin{array}{l}\text { Guadalupe River at U.S. Highway } 183 \text { near } \\
\text { Yoakum, Tex. }\end{array}$ & 32 & 27 & 08175800 & Guadalupe River at Cuero, Tex. & 4,934 \\
\hline & 26 & 08175000 & Sandies Creek near Westhoff, Tex. & 21 & & & & \\
\hline 23 & 27 & 08175800 & Guadalupe River at Cuero, Tex. & 53 & 28 & 08176500 & Guadalupe River at Victoria, Tex. & 5,198 \\
\hline 24 & -- & -- & -- & 22 & 29 & 08176550 & Fifteenmile Creek near Weser, Tex. & 167 \\
\hline 25 & 29 & 08176550 & Fifteenmile Creek near Weser, Tex. & 14 & 30 & 08176900 & $\begin{array}{l}\text { Coleto Creek at Arnold Road Crossing near } \\
\text { Schroeder, Tex. }\end{array}$ & 357 \\
\hline 26 & -- & -- & -- & 8.4 & 31 & 08177300 & Perdido Creek at FM 622 near Fannin, Tex. & 28 \\
\hline \multirow[t]{2}{*}{27} & 30 & 08176900 & $\begin{array}{l}\text { Coleto Creek at Arnold Road Crossing near } \\
\text { Schroeder, Tex. }\end{array}$ & 14 & 32 & 08177500 & Coleto Creek near Victoria, Tex. & 500 \\
\hline & 31 & 08177300 & Perdido Creek at FM 622 near Fannin, Tex. & 15 & & & & \\
\hline \multirow[t]{2}{*}{28} & 28 & 08176500 & Guadalupe River at Victoria, Tex. & 24 & 33 & 08177520 & Guadalupe River near Bloomington, Tex. & 5,816 \\
\hline & 32 & 08177500 & Coleto Creek near Victoria, Tex. & 19 & & & & \\
\hline \multirow[t]{2}{*}{29} & 33 & 08177520 & Guadalupe River near Bloomington, Tex. & 18 & 35 & 08188800 & Guadalupe River near Tivoli, Tex. & 10,128 \\
\hline & 34 & 08188570 & San Antonio River near McFaddin, Tex. & 14 & & & & \\
\hline
\end{tabular}


Table 3. Daily streamflow statistics for water years 1987-2011 for selected U.S. Geological Survey streamflow-gaging stations in the lower Guadalupe River Basin, southcentral Texas.

[USGS, U.S. Geological Survey; a water year is the 12-month period from October 1 through September 30 designated by the calendar year in which it ends; mi², square miles; $\mathrm{ft}^{3} / \mathrm{s}$, cubic feet per second; ,-- not applicable]

\begin{tabular}{|c|c|c|c|c|c|c|c|c|}
\hline $\begin{array}{l}\text { USGS } \\
\text { station } \\
\text { number }\end{array}$ & USGS station name & $\begin{array}{c}\text { Available } \\
\text { record } \\
\text { (water years) }\end{array}$ & $\begin{array}{c}\text { Drainage } \\
\text { area } \\
\left(\mathrm{mi}^{2}\right)\end{array}$ & $\begin{array}{c}\text { Daily mean } \\
\text { streamflow } \\
\left(\mathrm{ft}^{3} / \mathbf{s}\right)\end{array}$ & $\begin{array}{c}20 \text { percent } \\
\text { exceedance } \\
\text { streamflow } \\
\left(\mathrm{ft}^{3} / \mathrm{s}\right)\end{array}$ & $\begin{array}{c}50 \text { percent } \\
\text { exceedance } \\
\text { streamflow } \\
\left(\mathrm{ft}^{3} / \mathrm{s}\right)\end{array}$ & $\begin{array}{c}80 \text { percent } \\
\text { exceedance } \\
\text { streamflow } \\
\left(\mathrm{ft}^{3} / \mathrm{s}\right)\end{array}$ & $\begin{array}{c}90 \text { percent } \\
\text { exceedance } \\
\text { streamflow } \\
\left(\mathrm{ft}^{3} / \mathrm{s}\right)\end{array}$ \\
\hline 08167800 & Guadalupe River at Sattler, Tex. & 1960-2011 & 1,436 & 567 & 580 & 204 & 106 & 71.3 \\
\hline 08168500 & Guadalupe River above Comal River at New Braunfels, Tex. & $1928-2011$ & 1,518 & 662 & 736 & 267 & 131 & 88.5 \\
\hline 08168710 & Comal Springs at New Braunfels, Tex. & 1928-2011 & -- & 306 & 376 & 312 & 233 & 185 \\
\hline 08169000 & Comal River at New Braunfels, Tex. & $1928-2011$ & 130 & 325 & 384 & 315 & 234 & 187 \\
\hline 08170000 & San Marcos Springs at San Marcos, Tex. & 1956-2011 & -- & 185 & 239 & 171 & 115 & 100 \\
\hline 08171000 & Blanco River at Wimberley, Tex. & $1924-2011$ & 355 & 186 & 201 & 68.0 & 25.0 & 16.0 \\
\hline 08171300 & Blanco River near Kyle, Tex. & 1956-2011 & 412 & 182 & 200 & 47.0 & 5.00 & no flow \\
\hline 08172000 & San Marcos River at Luling, Tex. & 1939-2011 & 838 & 497 & 577 & 231 & 118 & 100 \\
\hline 08172400 & Plum Creek at Lockhart, Tex. & 1959-2011 & 112 & 49.4 & 18.0 & 1.10 & no flow & no flow \\
\hline 08173000 & Plum Creek near Luling, Tex. ${ }^{1}$ & $1930-2011$ & 309 & 120 & 59.0 & 10.0 & 2.56 & 2.56 \\
\hline 08173900 & Guadalupe River at Gonzales, Tex. ${ }^{1}$ & $1997-2011$ & 3,490 & 1,830 & 2,080 & 938 & 533 & 408 \\
\hline 08174600 & Peach Creek below Dilworth, Tex. ${ }^{1}$ & 1959-2011 & 460 & 146 & 34 & 6.75 & 2.00 & 1.20 \\
\hline 08175000 & Sandies Creek near Westhoff, Tex. & 1930-2011 & 549 & 144 & 33.5 & 8.60 & 2.70 & 1.43 \\
\hline 08175800 & Guadalupe River at Cuero, Tex. & $1964-2011$ & 4,934 & 2,220 & 2,340 & 1,010 & 554 & 424 \\
\hline 08176500 & Guadalupe River at Victoria, Tex. & $1935-2011$ & 5,198 & 2,340 & 2,460 & 1,070 & 566 & 426 \\
\hline 08176900 & Coleto Creek at Arnold Road Crossing near Schroeder, Tex. & 1979-2011 & 357 & 82.2 & 31.9 & 8.20 & 1.70 & 0.25 \\
\hline 08177500 & Coleto Creek near Victoria, Tex. & $1939-2011$ & 500 & 111 & 7.40 & 4.66 & 2.33 & 1.90 \\
\hline
\end{tabular}

${ }^{1}$ Missing record estimated using maintenance of variance extension type 1 (MOVE.1) (Hirsch, 1982) to calculate statistics for 1987-2011 period.

Stations with missing record during water years 1987-2011 and stations used in MOVE.1 computations to estimate missing record in the lower Guadalupe River Basin, south-central Texas.

\begin{tabular}{|c|c|c|c|}
\hline Streamflow-gaging station with missing record & Streamflow-gaging station from which daily mean streamflow obtained & Correlation coefficient & $\begin{array}{c}\text { Concurrent } \\
\text { values }\end{array}$ \\
\hline 08173000 Plum Creek near Luling, Tex. & 08172400 Plum Creek at Lockhart, Tex. & 0.86 & 6,284 \\
\hline 08173900 Guadalupe River at Gonzales, Tex. & 08175800 Guadalupe River at Cuero, Tex. & 0.95 & 5,478 \\
\hline 08174600 Peach Creek below Dilworth, Tex. & 08175000 Sandies Creek near Westhoff, Tex. & 0.82 & 4,017 \\
\hline
\end{tabular}

08174600 Peach Creek below Dilworth, Tex.

08175800 Guadalupe River at Cuero, Tex.

$0.82 \quad 4,017$ 
Three stations listed in table 3 had periods of missing record during water years 1987-2011 because of discontinuous streamflow-gaging station operation. Statistics in table 3 were computed by including estimates of missing daily streamflow record during the 25 -year period when data were not collected. Those estimates were calculated using an implementation of the maintenance of variance extension type 1 (MOVE.1) method (Hirsch, 1982) described in Granato (2009) with data from nearby stations (table 3). Record extension and gap filling were only applied if the coefficient of determination $\left(\mathrm{r}^{2}\right)$ (Helsel and Hirsch, 2002) between the streamflow-gaging station with missing data and a nearby streamflow-gaging station without missing data was greater than 0.8 , and there were more than 3,650 concurrent daily streamflow values (10 years).

Daily average per month inflows and outflows were provided by the Texas Commission for Environmental Quality (table 4, Michael Beatty, written commun., 2011; table 5, Angela Sander, written commun., 2011) and the U.S. Environmental Protection Agency (2013). Inflows consist primarily of treated wastewater-treatment plant discharges (table 4). Outflows consist primarily of withdrawals for public water supply. Inflows and outflows were assigned to the appropriate stream reach and included in the gain and loss calculations. Daily average per month outflow is defined as "The arithmetic average of all determinations of the daily discharge within a period on one calendar month" (U.S. Environmental Protection Agency, 2004).

Streamflow data were obtained from 31 continuous USGS streamflow-gaging stations, including 6 partial-record stations established to collect data for this study. These stations were operated to collect streamflow data for the range of flows typical of base-flow periods. Three 7-day base-flow periods were selected for assessment: March 15-21, 2010, April 10-16, 2011, and August 19-25, 2011. The primary selection criteria for the base-flow periods included the following considerations: (1) the streamflow was in a relatively steady state, that is, inflow to the lower Guadalupe River Basin was not affected by storm runoff, (2) desire to identify three periods with different streamflow and climatic conditions, and (3) streamflows were relatively small, compared with median streamflows. Because of interest in streamflow losses in the Guadalupe River between Oak Forest, Tex. (08169840), and Gonzalez, Tex. (08173900), an additional analysis was conducted for reaches 6, 7, 16, and 17 from September 22-28, 2012 (table 2).

Table 4. Inflow sites used for gain and loss computations in the lower Guadalupe River Basin, south-central Texas.

[EPA, U.S. Environmental Protection Agency; WWTP, wastewater-treatment plant]

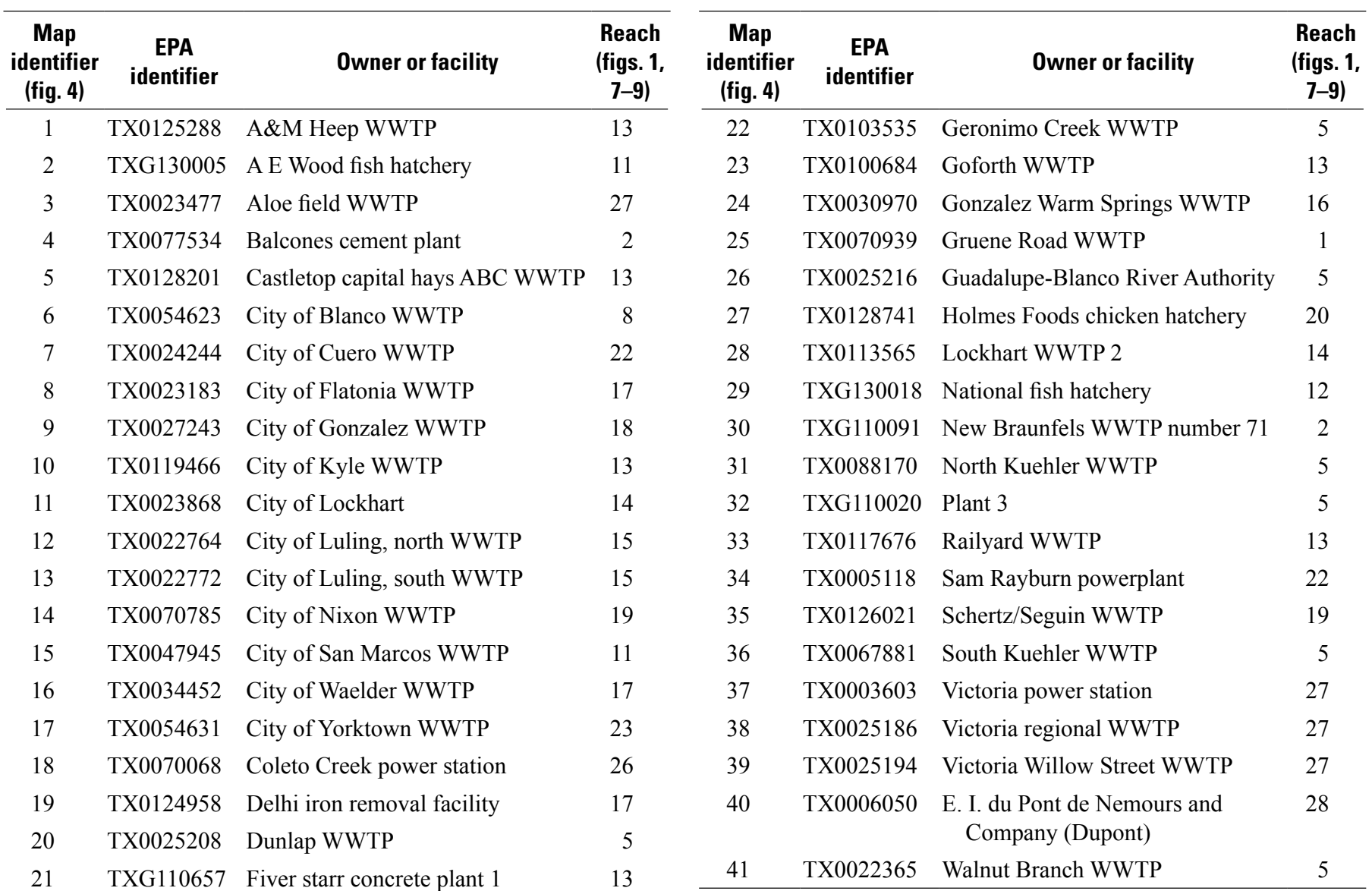


Table 5. Outflow sites used for gain and loss computations in the lower Guadalupe River Basin, south-central Texas.

\begin{tabular}{|c|c|c|}
\hline $\begin{array}{l}\text { Map } \\
\text { identi- } \\
\text { fier } \\
\text { (fig. 5) }\end{array}$ & $\begin{array}{l}\text { Name of entity or individual } \\
\text { responsible for reporting outflows } \\
\text { at the outflow site to the Texas } \\
\text { Commission for Environmental Quality }\end{array}$ & $\begin{array}{c}\text { Reach } \\
\text { (figs. 1, } \\
7-9 \text { ) }\end{array}$ \\
\hline 1 & Guadalupe-Blanco River Authority & 5 \\
\hline 2 & Robert M Kiehn & 16 \\
\hline 3 & San Marcos River Ranch, Limited & 13 \\
\hline 4 & City of New Braunfels & 3 \\
\hline 5 & New Braunfels Utilities & 3 \\
\hline 6 & Canyon Regional Water Authority & 6 \\
\hline 7 & Canyon Regional Water Authority & 6 \\
\hline 8 & Seguin Municipal Utilities & 5 \\
\hline 9 & Sara Darilek Rainwater & 5 \\
\hline 10 & City of Gonzales & 17 \\
\hline 11 & City of Gonzales & 17 \\
\hline 12 & King Ranch, Incorporated & 22 \\
\hline 13 & $\begin{array}{l}\text { South Texas Electric Cooperative, } \\
\text { Incorporated }\end{array}$ & 23 \\
\hline 14 & $\begin{array}{l}\text { E. I. du Pont de Nemours and Company } \\
\text { (Dupont) }\end{array}$ & 28 \\
\hline 15 & Texas Parks and Wildlife Department & 12 \\
\hline 16 & City of Blanco & 8 \\
\hline 17 & Canyon Regional Water Authority & 12 \\
\hline 18 & Barbara Baugh & 12 \\
\hline 19 & Tri Community Water Supply Corporation & 12 \\
\hline 20 & City of Luling & 13 \\
\hline 21 & Spencewood, Incorporated & 14 \\
\hline 22 & John Scott Greene & 12 \\
\hline 23 & Guadalupe-Blanco River Authority & 13 \\
\hline 24 & City of Victoria & 28 \\
\hline 25 & Victoria WLE LP ${ }^{1}$ & 28 \\
\hline 26 & Coleto Creek Power & 27 \\
\hline
\end{tabular}

${ }^{1}$ Official company name; combinations of letters that do not form words are part of the official name and are not acronyms.
For this report, a stream reach is defined as a stream channel extending from a downstream streamflow-gaging station to either the headwaters (defined as having no flow) or one or more upstream streamflow-gaging stations (fig. 1; table 2). Whereas each reach has a single downstream gaging station, branching (when present) of the stream within the reach at times resulted in multiple upstream gaging stations. Streamflow gains and losses were estimated by computing the difference in streamflow between the upstream and downstream ends of a reach minus any outflows from the reach plus any inflows into the reach. Streamflows at the upstream and downstream ends of each reach were determined from the continuous streamflow record of the streamflowgaging station(s) defining each stream reach (table 2). Because daily regulation (for power generation) affects streamflow in much of the study area, streamflows used for computing gains and losses were not solely based on discrete measurements.

Additional sources of gains or losses in a reach that were not specifically accounted for included evaporation from streams, groundwater inflow or outflow through the streambed, and unknown inflows and outflows. Streamflow gain or loss in a reach was computed as:

$$
G=Q_{D}-\Sigma Q_{U}+Q_{O}-Q_{I}
$$

where

$$
\begin{aligned}
G \quad= & \text { streamflow gain or loss; } \\
Q_{D} \quad= & \text { streamflow at the downstream streamflow- } \\
& \text { gaging station; }
\end{aligned}
$$

$\sum Q_{U} \quad=$ sum of streamflow at all upstream streamflow-gaging stations;

$Q_{O} \quad=$ outflows from the reach; and

$Q_{I} \quad=$ inflows into the reach

(Units of all variables in cubic feet per second).

When defined in this manner, positive values of $G$ indicate streamflow gains in a stream reach, whereas negative values indicate streamflow losses. The downstream and sum of the upstream streamflow values $\left(Q_{D}\right.$ and $\Sigma Q_{U}$, equation 1 , respectively) were based on the average of instantaneous streamflow computed every 15 minutes at the upstream and downstream streamflow-gaging stations of each reach during each 7-day base-flow period (March 15-21, 2010, April 10-16, 2011, and August 19-25, 2011). Outflows and inflows $\left(Q_{O}\right.$ and $Q_{V}$, respectively, in equation 1$)$ were based on monthly average (U.S. Environmental Protection Agency, 2004) inflows (table 4; Michael Beatty, Texas Commission on Environmental Quality, written commun., 2011) and outflows (table 5; Angela Sander, Texas Commission on Environmental Quality, written commun., 2011; U.S. Environmental Protection Agency, 2013). The locations of facilities providing inflow data are shown in figure 4 . The locations of permitted water rights are shown in figure 5 . 


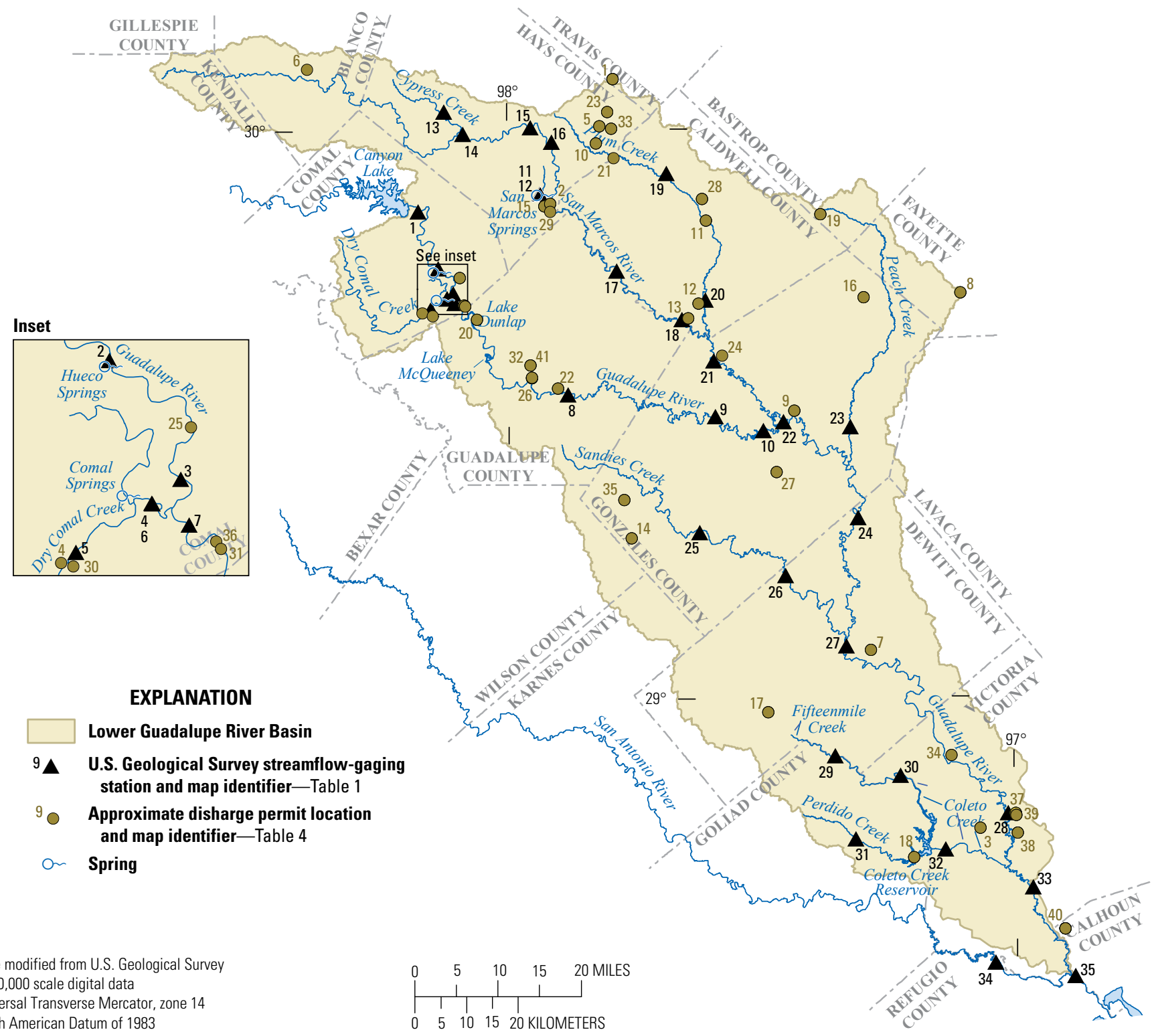

Figure 4. Location of inflow sites and U.S. Geological Survey streamflow-gaging stations used in the lower Guadalupe River Basin gain and loss study. 


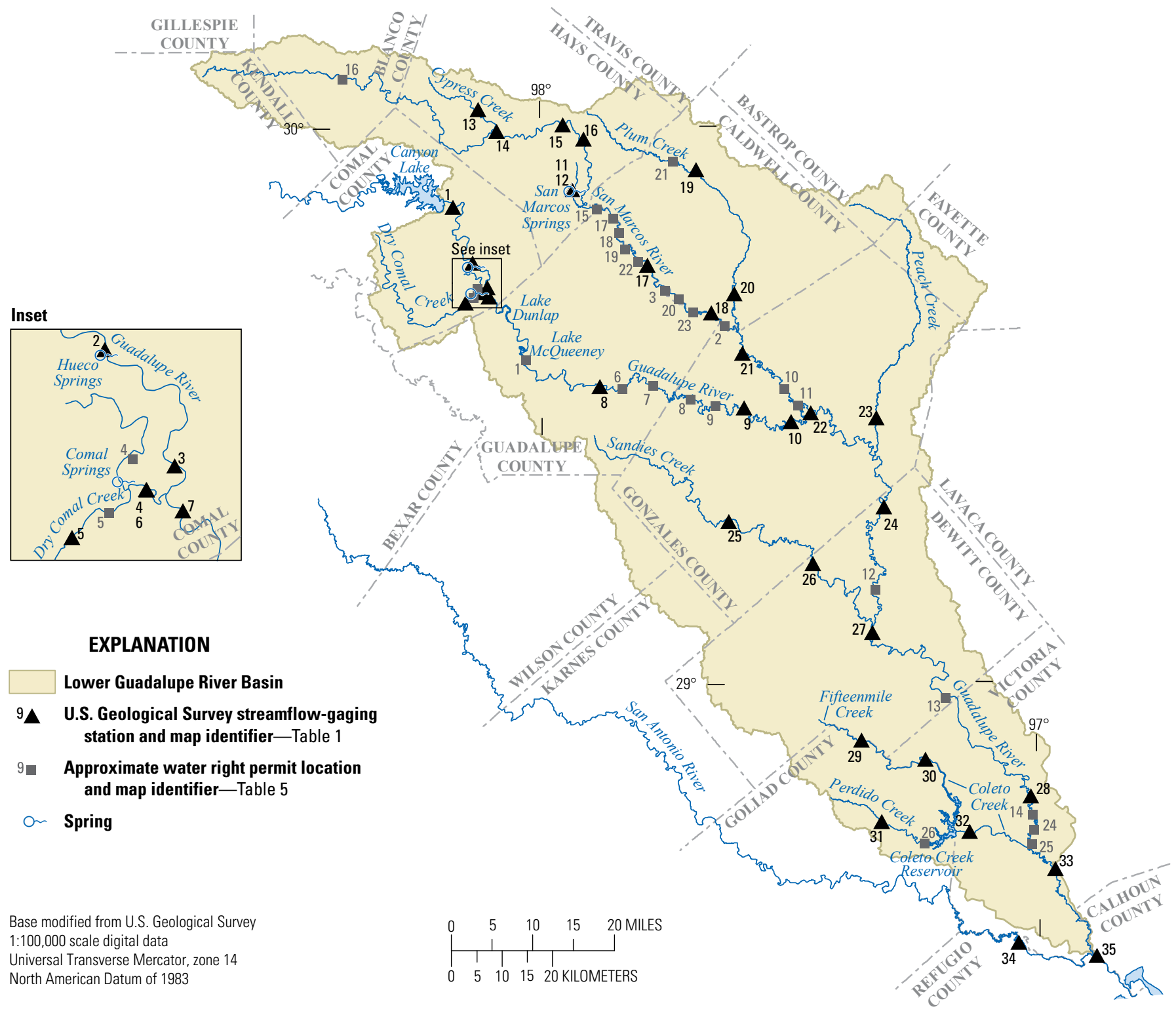

Figure 5. Water right permit locations and U.S. Geological Survey streamflow-gaging stations, lower Guadalupe River Basin, southcentral Texas. 
For the three 7-day base-flow periods (table 6) during March 2010, April 2011, and August 2011, the starting and ending dates for each period were chosen retrospectively to maximize the total number of field measurements made by hydrologic technicians within the period, thereby minimizing the uncertainty (error) of the upstream and downstream computed streamflows used for gain and loss computations. If two or more 7-day intervals had the same maximum number of field measurements, the interval that minimized streamflow variability, as measured by the daily mean coefficient of variation $(\mathrm{CV})$, was selected. The $\mathrm{CV}$ is the standard deviation of a dataset divided by the mean (Ott, 1988) and is a measure of the variability of the data. Table 6 lists the CV associated with base-flow periods in 2010 and 2011 selected for computing streamflow gains and losses. $\mathrm{CV}$ was not determined for the September 2012 base-flow assessment, which was made for a short reach of the river.

Streamflow at the downstream streamflow-gaging station and outflows and inflows $\left(Q_{D}, Q_{O}\right.$, and $Q_{T}$, respectively, equation 1) were computed as the arithmetic mean of all 15-minute instantaneous streamflow values during each 7-day base-flow period. To account for the effects of unsteady streamflow, traveltime was estimated to account for the elapsed time it takes for water to travel from each upstream gage of a reach to the downstream gage. To estimate traveltimes, stream velocities were determined for each upstream and downstream streamflow-gaging station pair of a reach. When one or more discharge measurements were available for a streamflow-gaging station during a base-flow period, the field-measured stream velocity with the highest accuracy rating was used. For each measurement rating the error (estimated difference between measured and total

Table 6. Base-flow periods in 2010 and 2011 selected for computing streamflow gains and losses in the lower Guadalupe River Basin, south-central Texas.

[CV, coefficient of variation]

\begin{tabular}{ccccc}
\hline $\begin{array}{c}\text { Base- } \\
\text { flow } \\
\text { period }\end{array}$ & Starting date ${ }^{1}$ & Ending date & $\begin{array}{c}\text { Total } \\
\text { number } \\
\text { of stream- } \\
\text { flow- } \\
\text { discharge } \\
\text { measure- } \\
\text { ments }\end{array}$ & $\begin{array}{c}\text { Mean daily } \\
\text { streamflow } \\
\text { variability } \\
\text { (CV }^{2} \\
\text { dimen- } \\
\text { sionless) }\end{array}$ \\
\hline 1 & March 15, 2010 & March 21, 2010 & 14 & 21 \\
2 & April 10, 2011 & April 16, 2011 & 16 & 19 \\
3 & August 19, 2011 & August 25, 2011 & 30 & 16 \\
\hline
\end{tabular}

${ }^{1}$ Starting and ending dates are associated with the downstream end of each stream reach. Streamflow hydrographs at the upstream end of each stream reach precede those of the downstream end of the reach to account for streamflow traveltime within each reach.

${ }^{2}$ The coefficient of variation is the standard deviation of a dataset divided by the mean (Ott, 1988). discharge) ranges are (1) less than or equal to 2 percent, excellent; (2) more than 2 and less than 5 percent, good; (3) greater than or equal to 5 and less than 8 percent, fair; and (4) equal to or more than 8 percent, poor (Fulford, 1992; Turnipseed and Sauer, 2010). The accuracy rating assigned by the hydrologic technician is based on factors such as cross-section uniformity, velocity homogeneity, streambed conditions, and other factors that affect the accuracy of each streamflow measurement (Turnipseed and Sauer, 2010). If more than one discharge measurement had the same (highest) accuracy rating, the mean of the highest rated field-measured stream velocities was used. If no discharge measurements were made at a streamflow-gaging station during a baseflow period, velocity was determined from the most recent measurement within 5 percent of the period streamflow; if no discharge measurements were within 5 percent, the measurement closest in value to average streamflow of the base-flow period was selected. The effective traveltime for an upstream and downstream streamflow-gaging station pair was computed as:

$$
T=D / V_{M E A N}
$$

where

$$
\begin{array}{cc}
T & =\text { effective traveltime between upstream and } \\
& \text { downstream gages, in hours; } \\
D & \text { distance between upstream and downstream } \\
& \text { gages, in miles; } \\
V_{\text {MEAN }} \quad \text { mean of upstream and downstream flow } \\
\quad \text { velocities, in miles per hour. }
\end{array}
$$

Traveltime between each upstream and downstream station was accounted for by shifting the base-flow period in time at the upstream station by the effective traveltime. This procedure was applied individually for each upstream and downstream pair of streamflow-gaging stations in a given reach. The effects of storage were not considered in the computation of traveltime.

To test the sensitivity of traveltime on computed discharge and gains or losses, traveltime $(T)$ for reach 9 on the Blanco River during the March 15-21, 2010, base-flow period, was varied by -50 percent and +50 percent. For reach 9, $T$ was estimated to be 19 hours during the March 15-21, 2010, base-flow period. For values of $T$ of 9.5 and 28.5 hours, the flow of the downstream streamflow-gaging station (08171290) was within +1.2 and +2.1 percent, respectively, of the streamflow computed for $T=19$ hours. The associated gain in streamflow for $T=19$ hours was $28 \mathrm{ft}^{3} / \mathrm{s}$. For values of $T=9.5$ and 28.5 hours, the gain in streamflow would be 25 and $23 \mathrm{ft}^{3} / \mathrm{s}$, respectively. The gains of 25 and $23 \mathrm{ft}^{3} / \mathrm{s}$ are within -11 and -18 percent, respectively, of the gain computed for $T=19$ hours.

Streamflow uncertainties were assigned to the upstream and downstream flows used in equation 1 according to the accuracy rating reported in the USGS annual water data report for that streamflow-gaging station (U.S. Geological Survey, 2012c). According to this scale, ratings of excellent, good, 
fair, and poor were given progressively increasing percentage uncertainties of $5,10,15$, and more than 15 percent, respectively. Percentage uncertainty estimates using the annual water data report rating were based on Novak (1985), which states that 95 percent of the time an excellent rating corresponds to less than or equal to 5 percent error, a good rating corresponds to less than or equal to 10 percent error, a fair rating corresponds to less than or equal to 15 percent error, and a poor rating indicates less than fair accuracy.

Streamflow uncertainties, in units of cubic feet per second, were computed by multiplying the appropriate percentage uncertainty by the streamflow value used in the gain and loss computation. The uncertainties associated with downstream and upstream streamflows of each reach were summed to obtain a composite streamflow-measurement uncertainty (Turco and others, 2007). The uncertainties of within-reach outflows and inflows, $Q_{O}$ and $Q_{P}$, were not evaluated.

For the studies conducted in 2010 and 2011, factors including evaporation, groundwater inflow or outflow through the streambed, and unknown withdrawals and return flows were not evaluated during the three baseflow periods. Evaporation from stream channels was not accounted for in the determination of streamflow gains and losses, but evaporation estimates from Lake Dunlap and Lake McQueeney (reach 5, fig. 1), downstream from New Braunfels, and Coleto Creek Reservoir (reach 27, fig. 1) are presented. Estimates of evaporation at these lakes were based on the surface area of each lake and monthly evaporation values published by the Texas Water Development Board (2013). Evaporation from Lake Dunlap was estimated as 2.1, 3.1, and $4.6 \mathrm{ft}^{3} / \mathrm{s}$ during March 2010, April 2011, and August 2011, respectively. Evaporation from Lake McQueeney was estimated as 2.0,3.0, and $4.5 \mathrm{ft}^{3} / \mathrm{s}$ during March 2010, April 2011, and August 2011, respectively. Evaporation from Coleto Creek Reservoir was estimated as 16,23 , and $29 \mathrm{ft}^{3} / \mathrm{s}$ during March 2010, April 2011, and August 2011, respectively.

For streamflow gains and losses computed in the September 22-28, 2012, base-flow period, groundwater inflows and outflows from the streambed and unknown withdrawals and return flows were not directly measured, but Penman's formula for evaporation from an open-water surface (Penman, 1948) was used to estimate evaporation from the stream channel for a similar period in 2011. For those computations, average air temperature and wind speed measured at a nearby weather station in New Braunfels, Tex., were acquired from the National Weather Service (National Oceanic and Atmospheric Administration, 2012). The average air temperature for selected periods was converted from degrees Celsius to atmospheric vapor pressure, in millimeters of mercury, using the relation between temperature and water vapor pressure (Oklahoma State University Chemistry
Department, 2001). Hourly water temperature data from USGS streamflow-gaging station 08188060 San Antonio River near Runge, Tex., also were used (U.S. Geological Survey, 2012a). The open-water surface area of that 14-mi reach of the Guadalupe River was calculated in ArcMap (Environmental Systems Research Institute, Inc., 2008) by using the editor function to create polygons on the river and summing of the areas of those polygons (about $0.497 \mathrm{mi}^{2}$ ). Evaporation losses from the Guadalupe River between Oak Forest, Tex., and H-5 dam for the weeks of August 12, 2011, August 19, 2011, and August 26, 2011, were 29.9, 51.2, and $8.4 \mathrm{ft}^{3} / \mathrm{s}$, respectively.

Possible influences of surficial geology (fig. 2) and aquifer outcrops (fig. 3) were considered in an evaluation of streamflow gains from or losses to groundwater. In calculating streamflow gains and losses, inflows and outflows were determined from daily average flow per month (U.S. Environmental Protection Agency, 2004).

Streamflows computed at Guadalupe-Blanco River Authority dams (Charlie Hickman, Guadalupe-Blanco River Authority, written commun., March 4, 2013) were not used in the computations of streamflow gains and losses; however, they qualitatively support the gain and loss survey. Comparisons to streamflows at the USGS streamflow-gaging stations downstream from each respective dam are presented. Nolte Dam is located about 4 mi upstream from USGS station 08169792 Guadalupe River at Farm Road 1117 near Seguin. The H-4 dam is about 2 mi upstream from 08169840 Guadalupe River at Oak Forest, Tex. The $\mathrm{H}-5$ dam is about 0.2 mi upstream from USGS station 08169860 Guadalupe River below the H-5 Dam near Gonzales, Tex. Flows computed for the dams during the March 2010 base-flow period were within 10 percent of the streamflows recorded by the respective nearest USGS streamflow-gaging stations. Flows computed for the dams computed for the April 2011 base-flow period were within 12 percent of the streamflows recorded by the respective nearest downstream USGS streamflow-gaging stations, except for the $\mathrm{H}-5$ dam, for which flow was 34 percent lower than the flow recorded at USGS station 08169860 . The reason for the difference in computed flows at the $\mathrm{H}-5$ dam and station 08169860 during the April 2011 base-flow period is not known but may be related to errors in the discharge ratings or to possible leakage under the dam. Flows computed for the dams during the August 2011 base-flow period were within 15 percent of the streamflows recorded by the respective nearest downstream USGS streamflow-gaging stations, except for Nolte Dam, for which flow was 21 percent higher than the streamflow recorded at USGS station 08169792. Flows computed for the $\mathrm{H}-4$ and $\mathrm{H}-5$ dams during the September 2012 base-flow period were within 18 percent of the streamflows of the respective USGS streamflow-gaging stations. 


\section{Streamflow Gains and Losses}

Streamflow gains and losses were computed for 21 reaches in the lower Guadalupe River Basin during March 15-21, 2010, April 10-16, 2011, and August 19-25, 2011, base-flow periods (tables 7-9, respectively), and for reaches 6 , 7, 16, and 17 near Gonzales, Tex., during the September 2012 base-flow period (table 10). Gains and losses are presented for each reach; the computed streamflow gain or loss per mile of stream reach are also presented to provide a sense of the relative magnitudes of gains or losses (tables 7-10). Only the gains or losses for the individual reaches are described. Streamflow gains or losses, which exceeded the sum of the associated streamflow uncertainties at the upstream and downstream ends of the reach (tables 7-10), are shown in figures 6-9.

Differences in the computed gains and losses during each base-flow period may be related to antecedent rainfall, stream levels, ground-water levels, evaporation, or other factors. The March 2010 base-flow period was preceded by a month of near normal rainfall (National Oceanic and Atmospheric Administration, 2013); the April and August and 2011 base-flow periods were marked by extreme rainfall deficits; and the September 2012 base-flow period was preceded by a month of below normal to near normal rainfall in the study area. Streamflows on the main-stem Guadalupe
River generally were well above normal during the March 2010 base-flow period, below normal during the April 2011 and September 2012 base-flow periods, and well below normal during the August 2011 base-flow period. The effects of drought on groundwater levels or evaporation during 2011 and 2012 have not been quantified as they relate to this study. Additionally, although each base-flow period represents a specific set of streamflow rates within the study area, the effects of factors such as air temperature, groundwater-level altitudes, or evapotranspiration were not considered in the analyses; computed gains or losses may not be representative of streamflow conditions during the associated periods.

The effects of undocumented withdrawals and unmeasured inflows on the streamflow gains and losses described in this report are unknown. In Texas, surface water belongs to the State, and a permit to withdraw water must be obtained from the Texas Commission on Environmental Quality (National Conference of State Legislatures, 2013). It is likely that not all withdrawals are permitted and documented, and these undocumented withdrawals might be an appreciable source of outflows (Wurbs and others, 1994). It is also likely that not all inflows contributing to streamflow in a reach could be measured; for example, in some reaches, unmeasured inflows from small streams and unmeasured irrigation return flows might represent an appreciable component of the streamflow. 
Table 7. Streamflow gains and losses computed for 21 stream reaches in the lower Guadalupe River Basin, south-central Texas, March 15-21, 2010.

[Streamflow gain values are positive and streamflow loss values are negative. Bold green font indicates a streamflow gain that exceeded the measurement uncertainty for this reach; USGS, U.S. Geological Survey; hr, hour; $\mathrm{ft}^{3} / \mathrm{s}$, cubic feet per second; mi, mile; --, data not available]

\begin{tabular}{|c|c|c|c|c|c|c|c|c|c|c|c|c|}
\hline $\begin{array}{l}\text { Reach } \\
\text { num- } \\
\text { ber }\end{array}$ & $\begin{array}{c}\text { Upstream } \\
\text { USGS } \\
\text { streamflow- } \\
\text { gaging station } \\
\text { number }\end{array}$ & $\begin{array}{l}\text { Map } \\
\text { identifier } \\
\text { for } \\
\text { upstream } \\
\text { station }^{1}\end{array}$ & $\begin{array}{l}\text { Downstream } \\
\text { USGS } \\
\text { streamflow- } \\
\text { gaging } \\
\text { station } \\
\text { number }\end{array}$ & $\begin{array}{c}\text { Map } \\
\text { identifier } \\
\text { for } \\
\text { down- } \\
\text { stream } \\
\text { station }\end{array}$ & $\begin{array}{c}\text { Estimated } \\
\text { traveltime ( }(T) \\
\text { between } \\
\text { upstream and } \\
\text { downstream } \\
\text { gaging stations } \\
\text { (hr) }\end{array}$ & $\begin{array}{c}\text { Upstream } \\
\text { streamflow } \\
\left(\mathrm{ft}^{3} / \mathrm{s}\right)\end{array}$ & $\begin{array}{c}\text { Down- } \\
\text { stream } \\
\text { streamflow } \\
\left(\mathrm{ft}^{3} / \mathbf{s}\right)\end{array}$ & $\begin{array}{c}\text { Estimated } \\
\text { outflow }{ }^{3} \\
\text { between } \\
\text { upstream } \\
\text { and down- } \\
\text { stream sites } \\
\left(\mathrm{ft}^{3} / \mathrm{s}\right)\end{array}$ & $\begin{array}{c}\text { Estimated } \\
\text { inflow } \\
\text { between } \\
\text { upstream and } \\
\text { downstream } \\
\text { sites } \\
\left(\mathrm{ft}^{3} / \mathrm{s}\right)\end{array}$ & $\begin{array}{c}\text { Stream- } \\
\text { flow gain } \\
\text { or loss } \\
\left(\mathrm{ft}^{3} / \mathrm{s}\right)\end{array}$ & $\begin{array}{l}\text { Streamflow } \\
\text { gain or } \\
\text { loss per } \\
\text { total reach } \\
\text { length } \\
{\left[\left(\mathrm{ft}^{3} / \mathrm{s}\right) / \mathrm{mi}\right]}\end{array}$ & $\begin{array}{c}\text { Streamflow } \\
\text { uncertainty } \\
\text { between } \\
\text { downstream } \\
\text { and upstream } \\
\text { sites } \\
\left(\mathrm{ft}^{3} / \mathrm{s}\right)\end{array}$ \\
\hline 1 & 08167800 & 1 & 08168500 & 3 & 10 & 529 & 660 & 0.0 & 0.72 & 130 & 8.67 & 119 \\
\hline 3 & 08168797 & 5 & 08169000 & 6 & 5 & 7.0 & 366 & 0.0 & 0.0 & 359 & 120 & 37 \\
\hline 4 & $\begin{array}{l}08168500 \\
08169000\end{array}$ & $\begin{array}{l}3 \\
6\end{array}$ & 08169500 & 7 & $\begin{array}{l}1 \\
2\end{array}$ & $\begin{array}{l}661 \\
366\end{array}$ & 999 & 0.0 & 0.0 & -28 & -7.57 & 203 \\
\hline 5 & 08169500 & 7 & 08169792 & 8 & 33 & 1,032 & 1,224 & 0.0 & 12.4 & 180 & 5.45 & 226 \\
\hline 6 & 08169792 & 8 & 08169840 & 9 & -- & 1,237 & -- & 0.0 & 0.0 & -- & -- & -- \\
\hline 7 & 08169840 & 9 & 08169860 & 10 & -- & -- & 1,187 & 0.0 & 0.0 & -- & -- & -- \\
\hline 9 & 08171000 & 14 & 08171290 & 15 & 19 & 242 & 270 & 0.0 & 0.0 & 28.0 & 2.33 & 65 \\
\hline 10 & 08171290 & 15 & 08171300 & 16 & 9 & 266 & 243 & 0.0 & 0.0 & -23.0 & -4.51 & 64 \\
\hline 12 & $\begin{array}{l}08170500 \\
08171300\end{array}$ & $\begin{array}{l}12 \\
16\end{array}$ & 08171500 & 17 & $\begin{array}{l}21 \\
43\end{array}$ & $\begin{array}{l}255 \\
233\end{array}$ & 554 & 0.00 & 8.84 & 57.2 & 1.00 & 156 \\
\hline 13 & 08171500 & 17 & 08172000 & 18 & 46 & 552 & 617 & 2.85 & 0.0 & 67.9 & 1.62 & 145 \\
\hline 15 & 08172400 & 19 & 08173000 & 20 & 34 & 23.9 & 45.4 & 0.0 & 1.70 & 19.8 & 0.86 & 10.4 \\
\hline 16 & $\begin{array}{l}08172000 \\
08173000\end{array}$ & $\begin{array}{l}18 \\
20\end{array}$ & 08173500 & 21 & $\begin{array}{l}15 \\
15\end{array}$ & $\begin{array}{l}616 \\
42.0\end{array}$ & 628 & 0.0 & 0.72 & -30.7 & -1.18 & 160 \\
\hline 17 & $\begin{array}{l}{ }^{4} 08169860 \\
08173500\end{array}$ & $\begin{array}{l}10 \\
21\end{array}$ & 08173900 & 22 & $\begin{array}{r}8 \\
17\end{array}$ & $\begin{array}{r}1,185 \\
619\end{array}$ & 1,941 & 2.41 & 0.0 & 139 & 3.76 & 465 \\
\hline 19 & $\begin{array}{l}08173900 \\
08174600\end{array}$ & $\begin{array}{l}22 \\
23\end{array}$ & 08174700 & 24 & $\begin{array}{l}23 \\
24\end{array}$ & $\begin{array}{r}1,943 \\
14.5\end{array}$ & 1,771 & 0.0 & 1.82 & -188 & -3.42 & 559 \\
\hline 21 & 08174970 & 25 & 08175000 & 26 & 60 & 14.5 & 23.9 & 0.0 & 0.0 & 9.4 & 0.39 & 4.6 \\
\hline 22 & $\begin{array}{l}08174700 \\
08175000\end{array}$ & $\begin{array}{l}24 \\
26\end{array}$ & 08175800 & 27 & $\begin{array}{l}36 \\
26\end{array}$ & $\begin{array}{r}1,772 \\
21.7\end{array}$ & 1,853 & 0.0 & 0.0 & 59.3 & 1.12 & 454 \\
\hline 23 & 08175800 & 27 & 08176500 & 28 & 54 & 1,868 & 1,831 & 0.0 & 1.53 & -38.5 & -0.73 & 370 \\
\hline 25 & 08176550 & 29 & 08176900 & 30 & -- & -- & -- & -- & -- & -- & -- & -- \\
\hline 27 & $\begin{array}{l}08176900 \\
08177300\end{array}$ & $\begin{array}{l}30 \\
31\end{array}$ & 08177500 & 32 & $\begin{array}{l}19 \\
37\end{array}$ & $\begin{array}{l}24.3 \\
0.58\end{array}$ & 41.1 & 0.0 & 0.0 & 16.2 & 0.56 & 7.8 \\
\hline 28 & $\begin{array}{l}08176500 \\
08177500\end{array}$ & $\begin{array}{l}28 \\
32\end{array}$ & 08177520 & 33 & $\begin{array}{l}27 \\
36\end{array}$ & $\begin{array}{r}1,815 \\
20.2\end{array}$ & 1,858 & 8.98 & 0.0 & 31.8 & 0.74 & 462 \\
\hline 29 & $\begin{array}{l}08177520 \\
08188570\end{array}$ & $\begin{array}{l}33 \\
34\end{array}$ & 08188800 & 35 & $\begin{array}{l}19 \\
18\end{array}$ & $\begin{array}{r}1,843 \\
657\end{array}$ & 2,792 & 0.0 & 0.0 & 292 & 9.13 & 794 \\
\hline
\end{tabular}

${ }^{1}$ Second map identifier is for the second station listed in the "Upstream USGS streamflow-gaging station number" column.

${ }^{2}$ Computed streamflow was adjusted for traveltime to minimize effects of nonsteady-state streamflow. Hydrograph at upstream station adjusted for traveltime, in hours, prior to that of downstream station.

${ }^{3}$ Monthly average values for March 2010 (Texas Commission on Environmental Quality, 2011; U.S. Environmental Protection Agency, 2013).

${ }^{4}$ Station 08169860 has no data during March 18-21. 
Table 8. Streamflow gains and losses computed for 21 stream reaches in the lower Guadalupe River Basin, south-central Texas, April 10-16, 2011.

[Streamflow gain values are positive and streamflow loss values are negative. Bold red font indicates a streamflow loss that exceeded the measurement uncertainty for this reach. Bold green font indicates a streamflow gain which exceeded the measurement uncertainty for this reach. USGS, U.S. Geological Survey; hr, hour; f $\mathrm{t}^{3} / \mathrm{s}$, cubic feet per second; mi, mile; --, data not available]

\begin{tabular}{|c|c|c|c|c|c|c|c|c|c|c|c|c|}
\hline $\begin{array}{c}\text { Reach } \\
\text { number }\end{array}$ & $\begin{array}{c}\text { Upstream } \\
\text { USGS } \\
\text { streamflow- } \\
\text { gaging } \\
\text { station } \\
\text { number }\end{array}$ & $\begin{array}{c}\text { Map } \\
\text { identifier } \\
\text { for } \\
\text { upstream } \\
\text { station' }\end{array}$ & $\begin{array}{c}\text { Downstream } \\
\text { USGS } \\
\text { streamflow- } \\
\text { gaging } \\
\text { station } \\
\text { number }\end{array}$ & $\begin{array}{l}\text { Map } \\
\text { identifier } \\
\text { for down- } \\
\text { stream } \\
\text { station }\end{array}$ & $\begin{array}{c}\text { Estimated } \\
\text { traveltime ( } T) \\
\text { between } \\
\text { upstream and } \\
\text { downstream } \\
\text { gaging stations } \\
\text { (hr) }\end{array}$ & $\begin{array}{c}\text { Upstream } \\
\text { streamflow } \\
\left(\mathrm{ft}^{3} / \mathrm{s}\right)\end{array}$ & $\begin{array}{c}\text { Down- } \\
\text { stream } \\
\text { streamflow } \\
\left(\mathrm{ft}^{3} / \mathbf{s}\right)\end{array}$ & $\begin{array}{l}\text { Estimated } \\
\text { outflow }{ }^{3} \text { be- } \\
\text { tween } \\
\text { upstream and } \\
\text { downstream } \\
\text { sites } \\
\left(\mathrm{ft}^{3} / \mathrm{s}\right)\end{array}$ & $\begin{array}{c}\text { Estimated } \\
\text { inflow } \\
\text { between } \\
\text { upstream and } \\
\text { downstream } \\
\text { sites } \\
\left(\mathrm{ft}^{3} / \mathrm{s}\right)\end{array}$ & $\begin{array}{c}\text { Stream- } \\
\text { flow gain } \\
\text { or loss } \\
\left(\mathrm{ft}^{3} / \mathbf{s}\right)\end{array}$ & $\begin{array}{c}\text { Streamflow } \\
\text { gain or } \\
\text { loss per } \\
\text { total reach } \\
\text { length } \\
{\left[\left(\mathrm{ft}^{3} / \mathrm{s}\right) / \mathrm{mi}\right]}\end{array}$ & $\begin{array}{c}\text { Streamflow } \\
\text { uncertainty } \\
\text { between } \\
\text { downstream } \\
\text { and upstream } \\
\text { sites } \\
\left(\mathrm{ft}^{3} / \mathrm{s}\right)\end{array}$ \\
\hline 1 & 08167800 & 1 & 08168500 & 3 & 35 & 54.4 & 96.0 & 0.00 & 0.91 & 40.7 & 2.71 & 15 \\
\hline 3 & 08168797 & 5 & 08169000 & 6 & 6 & 0.20 & 271 & 0.00 & 0.00 & 271 & 90.3 & 27 \\
\hline 4 & $\begin{array}{l}08168500 \\
08169000\end{array}$ & $\begin{array}{l}3 \\
6\end{array}$ & ${ }^{4} 08169500$ & 7 & $\begin{array}{l}2 \\
1\end{array}$ & $\begin{array}{l}96.2 \\
290\end{array}$ & 289 & 0.00 & 0.00 & -97.2 & -26.3 & 68 \\
\hline 5 & 08169500 & 7 & 08169792 & 8 & 24 & 292 & 326 & 0.00 & 13.9 & 20.1 & 0.61 & 62 \\
\hline 6 & 08169792 & 8 & 08169840 & 9 & -- & 344 & -- & 0.00 & 0.00 & -- & -- & -- \\
\hline 7 & 08169840 & 9 & 08169860 & 10 & -- & -- & 467 & 0.00 & 0.00 & -- & -- & -- \\
\hline 9 & 08171000 & 14 & 08171290 & 15 & 44 & 28.2 & 22.6 & 0.00 & 0.00 & -5.6 & -0.47 & 6.2 \\
\hline 10 & 08171290 & 15 & 08171300 & 16 & 17 & 23.0 & 4.3 & 0.00 & 0.00 & -18.7 & -3.67 & 3.9 \\
\hline 12 & $\begin{array}{l}08170500 \\
08171300\end{array}$ & $\begin{array}{l}12 \\
16\end{array}$ & ${ }^{4} 08171500$ & 17 & $\begin{array}{r}30 \\
102\end{array}$ & $\begin{array}{l}141 \\
6.84\end{array}$ & 155 & 0.00 & 6.96 & 7.16 & 0.13 & 45 \\
\hline 13 & ${ }^{4} 08171500$ & 17 & 08172000 & 18 & 52 & 157 & 143 & 0.00 & 0.00 & -14.0 & -0.33 & 38 \\
\hline 15 & 08172400 & 19 & 08173000 & 20 & 99 & 1.74 & 6.99 & 0.00 & 2.01 & 3.24 & 0.14 & 1.3 \\
\hline 16 & $\begin{array}{l}08172000 \\
08173000\end{array}$ & $\begin{array}{l}18 \\
20\end{array}$ & ${ }^{4} 08173500$ & 21 & $\begin{array}{l}19 \\
44\end{array}$ & $\begin{array}{l}144 \\
7.50\end{array}$ & 157 & 0.32 & 0.71 & 5.11 & 0.20 & 39 \\
\hline 17 & $\begin{array}{l}08169860 \\
08173500\end{array}$ & $\begin{array}{l}10 \\
21\end{array}$ & 08173900 & 22 & $\begin{array}{l}10 \\
74\end{array}$ & $\begin{array}{l}443 \\
161\end{array}$ & 449 & 0.00 & 0.00 & -155 & -4.19 & 136 \\
\hline 19 & $\begin{array}{l}08173900 \\
08174600\end{array}$ & $\begin{array}{l}22 \\
23\end{array}$ & ${ }^{4} 08174700$ & 24 & $\begin{array}{l}80 \\
72\end{array}$ & $\begin{array}{l}490 \\
1.87\end{array}$ & 512 & 0.00 & 1.17 & 19.0 & 0.35 & 151 \\
\hline 21 & 08174970 & 25 & 08175000 & 26 & -- & -- & -- & -- & -- & -- & -- & -- \\
\hline 22 & $\begin{array}{l}08174700 \\
08175000\end{array}$ & $\begin{array}{l}24 \\
26\end{array}$ & 08175800 & 27 & $\begin{array}{l}67 \\
36\end{array}$ & $\begin{array}{l}500 \\
1.67\end{array}$ & 553 & 1.38 & 0.00 & 52.7 & 0.99 & 131 \\
\hline 23 & 08175800 & 27 & 08176500 & 28 & 95 & 588 & 503 & 0.00 & 1.58 & -86.6 & -1.63 & 134 \\
\hline 25 & 08176550 & 29 & 08176900 & 30 & -- & -- & -- & -- & -- & -- & -- & -- \\
\hline 27 & $\begin{array}{l}08176900 \\
08177300\end{array}$ & $\begin{array}{l}30 \\
31\end{array}$ & 08177500 & 32 & $\begin{array}{l}52 \\
82\end{array}$ & $\begin{array}{l}6.25 \\
0.03\end{array}$ & 5.15 & 0.00 & 0.00 & -1.13 & -0.04 & 1.5 \\
\hline 28 & $\begin{array}{l}08176500 \\
08177500\end{array}$ & $\begin{array}{l}28 \\
32\end{array}$ & 08177520 & 33 & $\begin{array}{l}50 \\
57\end{array}$ & $\begin{array}{l}519 \\
5.40\end{array}$ & 518 & 0.00 & 0.00 & -6.4 & -0.15 & 130 \\
\hline 29 & $\begin{array}{l}08177520 \\
08188570\end{array}$ & $\begin{array}{l}33 \\
34\end{array}$ & 08188800 & 35 & $\begin{array}{l}44 \\
33\end{array}$ & $\begin{array}{l}523 \\
266\end{array}$ & 731 & 0.00 & 0.00 & -58.0 & -1.81 & 228 \\
\hline
\end{tabular}

${ }^{1}$ Second map identifier is for the second station listed in the "Upstream USGS streamflow-gaging station number" column.

${ }^{2}$ Computed streamflow was adjusted for traveltime to minimize effects of nonsteady-state streamflow. Hydrograph at upstream station adjusted for traveltime, in hours, prior to that of downstream station.

${ }^{3}$ Monthly average values for April 2011 (Texas Commission on Environmental Quality, 2011; U.S. Environmental Protection Agency, 2013).

${ }^{4} 08169500$ and 08174700 have no data for April 16. 08171500 and 08173500 have no data for April 15-16. 
Table 9. Streamflow gains and losses computed for 21 stream reaches in the lower Guadalupe River Basin, south-central Texas, August $19-25,2011$.

[Streamflow gain values are positive and streamflow loss values are negative. Bold red font indicates a streamflow loss that exceeded the measurement uncertainty for this reach. Bold green font indicates a streamflow gain which exceeded the measurement uncertainty for this reach. USGS, U.S. Geological Survey; hr, hour; fts/s, cubic foot per second; mi, mile; N/A, not applicable; --, data not available]

\begin{tabular}{|c|c|c|c|c|c|c|c|c|c|c|c|c|}
\hline $\begin{array}{c}\text { Reach } \\
\text { Number }\end{array}$ & $\begin{array}{l}\text { Upstream } \\
\text { USGS } \\
\text { streamflow- } \\
\text { gaging sta- } \\
\text { tion } \\
\text { number }\end{array}$ & $\begin{array}{c}\text { Map } \\
\text { identifier } \\
\text { for } \\
\text { upstream } \\
\text { station' }^{1}\end{array}$ & $\begin{array}{l}\text { Downstream } \\
\text { USGS } \\
\text { streamflow } \\
\text { gaging } \\
\text { station } \\
\text { number }\end{array}$ & $\begin{array}{c}\text { Map } \\
\text { identifier } \\
\text { for } \\
\text { down- } \\
\text { stream } \\
\text { station }\end{array}$ & $\begin{array}{c}\text { Estimated } \\
\text { traveltime }(\pi) \\
\text { between upstream } \\
\text { and downstream } \\
\text { gaging stations } \\
\text { (hr) }\end{array}$ & $\begin{array}{c}\text { Upstream } \\
\text { streamflow } \\
\left(\mathrm{ft}^{3} / \mathrm{s}\right)\end{array}$ & $\begin{array}{c}\text { Down- } \\
\text { stream } \\
\text { streamflow } \\
\left(\mathrm{ft}^{3} / \mathbf{s}\right)\end{array}$ & $\begin{array}{c}\text { Estimated } \\
\text { outflow } \\
\text { between } \\
\text { upstream and } \\
\text { downstream } \\
\text { sites } \\
\left(\mathrm{ft}^{3} / \mathrm{s}\right)\end{array}$ & $\begin{array}{c}\text { Estimated } \\
\text { inflow } \\
\text { between } \\
\text { upstream } \\
\text { and down- } \\
\text { stream sites } \\
\left(\mathrm{ft}^{3} / \mathbf{s}\right)\end{array}$ & $\begin{array}{c}\text { Stream- } \\
\text { flow } \\
\text { gain or } \\
\text { loss } \\
\left(\mathrm{ft}^{3} / \mathbf{s}\right)\end{array}$ & $\begin{array}{c}\text { Streamflow } \\
\text { gain or loss } \\
\text { per total } \\
\text { reach length } \\
{\left[\left(\mathrm{ft}^{3} / \mathrm{s}\right) / \mathrm{mi}\right]}\end{array}$ & $\begin{array}{c}\text { Streamflow } \\
\text { uncertainty } \\
\text { between } \\
\text { downstream } \\
\text { and upstream } \\
\text { sites } \\
\left(\mathrm{ft}^{3} / \mathrm{s}\right)\end{array}$ \\
\hline 1 & 08167800 & 1 & 08168500 & 3 & 36 & 63.9 & 60.9 & 0.00 & 0.69 & -3.7 & -0.25 & 12 \\
\hline 3 & 08168797 & 5 & 08169000 & 6 & 9 & 0.00 & 168 & 0.00 & 0.00 & 168 & 56.0 & 17 \\
\hline 4 & $\begin{array}{l}08168500 \\
08169000\end{array}$ & $\begin{array}{l}3 \\
6\end{array}$ & 08169500 & 7 & $\begin{array}{l}2 \\
2\end{array}$ & $\begin{array}{l}60.8 \\
168\end{array}$ & 146 & 0.00 & 0.00 & -82.8 & -22.4 & 37 \\
\hline 5 & 08169500 & 7 & 08169792 & 8 & 26 & 147 & 106 & 0.39 & 12.9 & -53.5 & -1.62 & 25 \\
\hline 6 & 08169792 & 8 & 08169840 & 9 & -- & 130 & -- & 0.00 & 0.00 & -- & -- & -- \\
\hline 7 & 08169840 & 9 & 08169860 & 10 & -- & -- & 129 & 0.00 & 0.00 & -- & -- & -- \\
\hline 9 & 08171000 & 14 & 08171290 & 15 & 34 & 8.84 & 6.64 & 0.00 & 0.00 & -2.20 & -0.18 & 1.9 \\
\hline 10 & 08171290 & 15 & 08171300 & 16 & 18 & 6.60 & 0.00 & 0.00 & 0.00 & -6.60 & -1.29 & 1.0 \\
\hline 12 & $\begin{array}{l}08170500 \\
08171300\end{array}$ & $\begin{array}{l}12 \\
16\end{array}$ & 08171500 & 17 & $\begin{array}{c}29 \\
\text { N/A }\end{array}$ & $\begin{array}{c}93.7 \\
0.00\end{array}$ & 82.8 & 0.00 & 5.78 & -16.7 & -0.29 & 26 \\
\hline 13 & 08171500 & 17 & 08172000 & 18 & 67 & 84.3 & 73.7 & 0.00 & 0.00 & -10.6 & -0.25 & 20 \\
\hline 15 & 08172400 & 19 & 08173000 & 20 & 116 & 2.48 & 2.38 & 0.00 & 2.01 & -2.11 & -0.09 & 0.7 \\
\hline 16 & $\begin{array}{l}08172000 \\
08173000\end{array}$ & $\begin{array}{l}18 \\
20\end{array}$ & 08173500 & 21 & $\begin{array}{l}18 \\
25\end{array}$ & $\begin{array}{c}73.8 \\
2.72\end{array}$ & 78.9 & 0.00 & 0.67 & 1.71 & 0.07 & 19 \\
\hline 17 & $\begin{array}{l}08169860 \\
08173500\end{array}$ & $\begin{array}{l}10 \\
21\end{array}$ & 08173900 & 22 & $\begin{array}{l}13 \\
40\end{array}$ & $\begin{array}{l}131 \\
79.2\end{array}$ & 178 & 0.00 & 0.00 & -32.2 & -0.87 & 49 \\
\hline 19 & $\begin{array}{l}08173900 \\
08174600\end{array}$ & $\begin{array}{l}22 \\
23\end{array}$ & 08174700 & 24 & $\begin{array}{l}-- \\
--\end{array}$ & -- & -- & -- & -- & -- & -- & -- \\
\hline 21 & 08174970 & 25 & 08175000 & 26 & $\mathrm{~N} / \mathrm{A}^{4}$ & 0.00 & 0.092 & 0.00 & 0.00 & 0.092 & 0.004 & 0.01 \\
\hline 22 & $\begin{array}{l}08174700 \\
08175000\end{array}$ & $\begin{array}{l}24 \\
26\end{array}$ & 08175800 & 27 & -- & $\begin{array}{l}-- \\
--\end{array}$ & -- & -- & -- & -- & -- & -- \\
\hline 23 & 08175800 & 27 & 08176500 & 28 & 120 & 201 & 186 & 0.00 & 1.51 & -16.5 & -0.31 & 48 \\
\hline 25 & 08176550 & 29 & 08176900 & 30 & -- & -- & -- & -- & -- & -- & -- & -- \\
\hline 27 & $\begin{array}{l}08176900 \\
08177300\end{array}$ & $\begin{array}{l}30 \\
31\end{array}$ & 08177500 & 32 & $\begin{array}{l}\text { N/A }{ }^{4} \\
\text { N/A }\end{array}$ & $\begin{array}{l}0.00 \\
0.00\end{array}$ & 1.98 & 0.00 & 0.00 & 1.98 & 0.07 & 0.2 \\
\hline 28 & $\begin{array}{l}08176500 \\
08177500\end{array}$ & $\begin{array}{l}28 \\
32\end{array}$ & 08177520 & 33 & $\begin{array}{r}110 \\
98\end{array}$ & $\begin{array}{l}173 \\
1.88\end{array}$ & 176 & 0.00 & 0.00 & 1.1 & 0.03 & 44 \\
\hline 29 & $\begin{array}{l}08177520 \\
08188570\end{array}$ & $\begin{array}{l}33 \\
34 \\
\end{array}$ & 08188800 & 35 & $\begin{array}{l}109 \\
117\end{array}$ & $\begin{array}{c}184 \\
88.1\end{array}$ & 281 & 0.00 & 0.00 & 8.9 & 0.28 & 83 \\
\hline
\end{tabular}

${ }^{1}$ Second map identifier is for the second station listed in the "Upstream USGS streamflow-gaging station number" column.

${ }^{2}$ Computed streamflow was adjusted for traveltime to minimize effects of nonsteady-state streamflow. Hydrograph at upstream station adjusted for traveltime, in hours, prior to that of downstream station.

${ }^{3}$ Monthly average values for August 2011 (Texas Commission on Environmental Quality, 2011; U.S. Environmental Protection Agency, 2013).

${ }^{4}$ Traveltime not applicable because of zero streamflow at the upstream station. 
Table 10. Streamflow gains and losses computed for a stream reach on the Guadalupe River from Seguin, Texas, to Gonzales, Tex., September 22-28, 2012.

[Streamflow gain values are positive and streamflow loss values are negative. USGS, U.S. Geological Survey; hr, hour; fts/s, cubic feet per second; mi, mile; --, data not available]

\begin{tabular}{|c|c|c|c|c|c|c|c|c|c|c|c|c|}
\hline $\begin{array}{l}\text { Reach } \\
\text { number }\end{array}$ & $\begin{array}{l}\text { Upstream } \\
\text { USGS } \\
\text { streamflow- } \\
\text { gaging } \\
\text { station } \\
\text { number }\end{array}$ & $\begin{array}{c}\text { Map } \\
\text { identifier } \\
\text { for } \\
\text { upstream } \\
\text { station' }\end{array}$ & $\begin{array}{l}\text { Downstream } \\
\text { USGS } \\
\text { streamflow- } \\
\text { gaging } \\
\text { station } \\
\text { number }\end{array}$ & $\begin{array}{c}\text { Map } \\
\text { identifier for } \\
\text { downstream } \\
\text { station }\end{array}$ & $\begin{array}{c}\text { Estimated } \\
\text { traveltime ( } T) \\
\text { between upstream } \\
\text { and downstream } \\
\text { gaging stations } \\
\text { (hr) }\end{array}$ & $\begin{array}{c}\text { Upstream } \\
\text { streamflow } \\
\left(\mathrm{ft}^{3} / \mathbf{s}\right)\end{array}$ & $\begin{array}{c}\text { Down- } \\
\text { stream } \\
\text { stream- } \\
\text { flow } \\
\left(\mathrm{ft}^{3} / \mathrm{s}\right)\end{array}$ & $\begin{array}{c}\text { Estimated } \\
\text { outflow } \\
\text { between } \\
\text { upstream } \\
\text { and } \\
\text { downstream } \\
\text { sites } \\
\left(\mathrm{ft}^{3} / \mathbf{s}\right)\end{array}$ & $\begin{array}{c}\text { Estimated } \\
\text { inflow }{ }^{3} \\
\text { between } \\
\text { upstream } \\
\text { and } \\
\text { downstream } \\
\text { sites } \\
\left(\mathrm{ft}^{3} / \mathbf{s}\right)\end{array}$ & $\begin{array}{c}\text { Stream- } \\
\text { flow } \\
\text { gain or } \\
\text { loss } \\
\left(\mathrm{ft}^{3} / \mathbf{s}\right)\end{array}$ & $\begin{array}{l}\text { Stream- } \\
\text { flow gain } \\
\text { or loss per } \\
\text { total reach } \\
\text { length } \\
{\left[\left(\mathrm{ft}^{3} / \mathrm{s}\right) / \mathrm{mi}\right]}\end{array}$ & $\begin{array}{c}\text { Streamflow } \\
\text { uncertainty } \\
\text { between } \\
\text { downstream } \\
\text { and } \\
\text { upstream } \\
\text { sites } \\
\left(\mathrm{ft}^{3} / \mathrm{s}\right)\end{array}$ \\
\hline 6 & 08169792 & 8 & 08169840 & 9 & -- & 424 & - & 0.0 & 0.0 & -- & -- & -- \\
\hline 7 & 08169840 & 9 & 08169860 & 10 & -- & -- & 330 & 0.0 & 0.0 & -- & -- & -- \\
\hline $\begin{array}{r}16 \\
{ }^{4} 17\end{array}$ & $\begin{array}{l}08169860 \\
08172000 \\
08173000\end{array}$ & $\begin{array}{l}10 \\
18 \\
20\end{array}$ & 08173900 & 22 & $\begin{array}{l}10 \\
61 \\
71\end{array}$ & $\begin{array}{l}335 \\
158 \\
5.65\end{array}$ & 454 & 3.11 & 0.72 & -42 & -1.09 & 120 \\
\hline
\end{tabular}

${ }^{1}$ Second and third map identifiers are for the second and third stations, respectively, listed in the "Upstream USGS streamflow-gaging station number" column.

${ }^{2}$ Computed streamflow was adjusted for traveltime to minimize effects of nonsteady-state streamflow. Hydrograph at upstream station adjusted for traveltime, in hours, prior to that of downstream station.

${ }^{3}$ Monthly average values for September 2012 (Texas Commission on Environmental Quality, 2011; U.S. Environmental Protection Agency, 2013).

${ }^{4}$ Station 08173500 San Marcos River at Ottine, Tex., (site 21) was not active during 2012, so reaches 16 and 17 were combined for September 2012 analysis period. 


\section{Streamflow Gains and Losses during March 15-21, 2010}

Streamflow gains greater than the uncertainty in the computed streamflow were measured during a period of elevated base flow from March 15-21, 2010, in reaches 1, 3, 15, 21 and 27 (fig. 6; table 7). Reach 1 (fig. 1), on the Guadalupe River downstream from Canyon Lake, gained $130 \mathrm{ft}^{3} / \mathrm{s}$. Groundwater inflows from the Edwards aquifer account for much of this gain, with discharges from Hueco Springs representing a primary source of these inflows. The average daily streamflow at USGS streamflow-gaging station 08168000 Hueco Springs near New Braunfels, Tex., was $76.4 \mathrm{ft}^{3} / \mathrm{s}$ during March 15-21, 2010. Reach 3 on the Comal River gained $359 \mathrm{ft}^{3} / \mathrm{s}$, nearly all of which represents the inflow from Comal Springs. Reach 15 on Plum Creek between Lockhart and Luling gained $19.8 \mathrm{ft}^{3} / \mathrm{s}$, likely as inflows from the Carrizo-Wilcox aquifer (mostly from the Wilcox Group, the primary formation exposure of this aquifer in this reach), or from alluvial terrace deposits of the Leona Formation (figs. 2 and 3). Reach 21 on Sandies Creek gained $9.4 \mathrm{ft}^{3} / \mathrm{s}$, which might be from inflows from the Yegua Jackson aquifer, which is composed primarily of the Yegua Formation (fig. 3). Reach 27 gained $16.2 \mathrm{ft}^{3} / \mathrm{s}$; this gain is likely from groundwater inflows from the Gulf Coast aquifer, which are enhanced by seepage losses from Coleto Creek Reservoir that contribute to groundwater recharge from the Gulf Coast aquifer (the interaction of surface water and groundwater at Coleto Creek Reservoir). During the period of elevated base flow in March 2010, streamflow losses greater than the uncertainty in the computed streamflow were not measured in any of the reaches.

\section{Streamflow Gains and Losses during April 10-16, 2011}

During the April 10-16, 2011, base-flow period, reaches 1,3 , and 15 recorded gains greater than the uncertainty in the computed streamflow (fig. 7; table 8). The sources of these gains were believed to be the same as during the March 15-21 assessment of gains and losses for these reaches. Reach 1 on the Guadalupe River gained $40.7 \mathrm{ft}^{3} / \mathrm{s}$ (table 8). Reach 3 on the Comal River gained $271 \mathrm{ft}^{3} / \mathrm{s}$, which was similar to the gain of $359 \mathrm{ft}^{3} / \mathrm{s}$ recorded in this reach during March 15-21, 2010. Reach 15 on Plum Creek between Lockhart and Luling gained $3.24 \mathrm{ft}^{3} / \mathrm{s}$ (fig. 7). During this base-flow period, losses greater in magnitude than the uncertainty in the computed streamflow were measured in three reaches (fig. 7). Reach 4, on the Guadalupe River at New Braunfels, Tex., lost $97.2 \mathrm{ft}^{3} / \mathrm{s}$. There are no aquifer outcrops along reach 4 , and the reason for this loss is not known. Puente (1978, p. 28) made the following observations pertaining to the Guadalupe River immediately upstream from reach 4:

The Guadalupe River crosses the infiltration area of the Edwards aquifer [outcrop of the Edwards Formation, fig. 2 in this report], but does not contribute recharge in significant quantities. Although 48 square miles of area in the Guadalupe River basin is within the infiltration area, seepage studies indicate that the net streamflow losses and gains in the area are small and insignificant. The potentiometric surface of the aquifer in the New Braunfels area is generally at the level of the streambed of the Guadalupe River and is relatively stable because of the large and almost perennial flow of Comal Springs.

Puente (1978) was referring to the reach of the Guadalupe River that crosses the outcrop of the Edwards aquifer, and reach 4 begins at USGS streamflow-gaging stations 08168500 Guadalupe River above Comal River at New Braunfels, Tex (map identifier 3) and 08169000 Comal River at New Braunfels, Tex. (map identifier 6) - both of which are about 1 mi downstream from the outcrop of the Edwards aquifer. If the Guadalupe River does not provide appreciable recharge to the Edwards aquifer when it flows over the Edwards aquifer outcrop, it is unlikely to provide appreciable groundwater recharge downstream from the outcrop; however, according to the U.S. Drought Monitor (University of Nebraska-Lincoln, 2013), by April 2011, Comal, Guadalupe, and Gonzales Counties were in extreme drought. Hence, infiltration along the stream channel may have occurred in areas where this had not been previously documented. Reach 10, the Blanco River near Kyle, lost $18.7 \mathrm{ft}^{3} / \mathrm{s}$. Most of the losses in this reach are likely in the form of recharge to the Edwards aquifer, which occurs through the numerous faults intersecting the channel (fig. 7) (Hanson and Small, 1995; U.S. Geological Survey, 2012b) northwest of Kyle (fig. 1). Reach 17, which includes the confluence of the Guadalupe and San Marcos Rivers (fig. 7, inset B), lost $155 \mathrm{ft}^{3} / \mathrm{s}$. Recharge to the outcrops of Sparta, Queen City, and Yegua Jackson aquifers along this reach likely accounts for much of the streamflow losses that occur in this reach. 


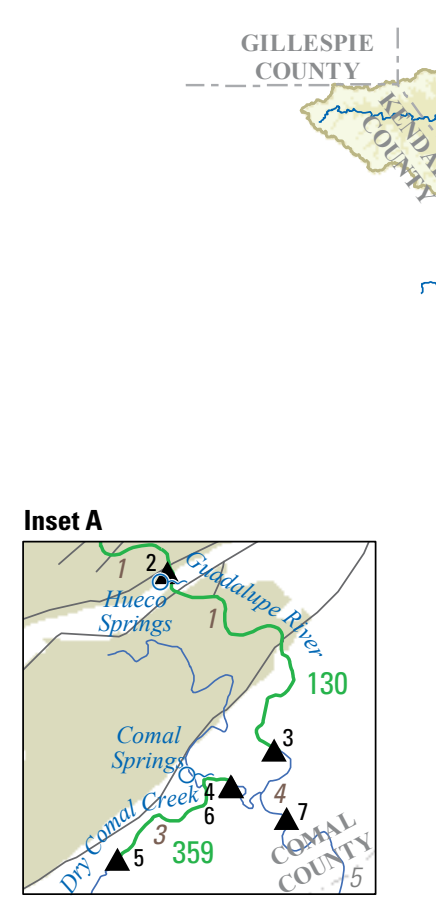

Inset B

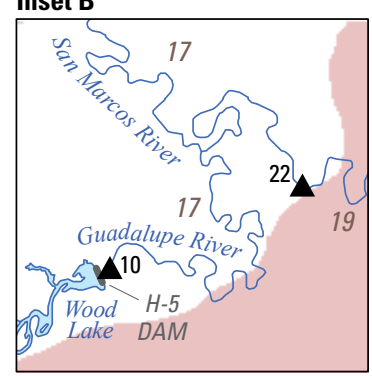

Base modified from U.S. Geological Survey 1:100,000 scale digital data

Universal Transverse Mercator, zone 14

North American Datum of 1983
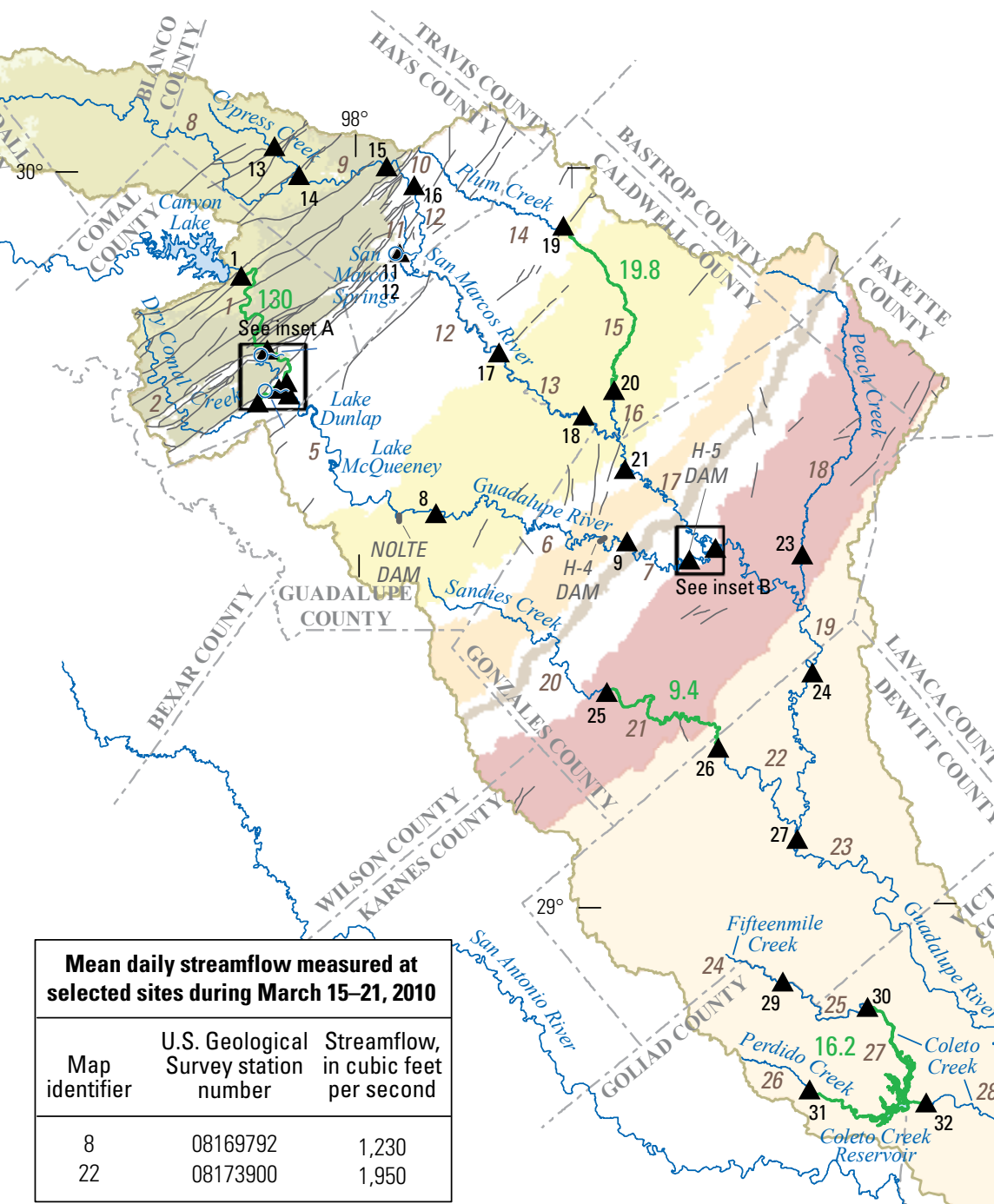

(2)

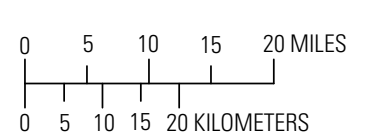

\section{EXPLANATION}

Major aquifer system

Gulf Coast

Carrizo-Wilcox

Edwards-Trinity

Edwards

Trinity

\section{Minor aquifer system}

Yegua Jackson

Sparta

Queen City

Fault

\section{Lower Guadalupe River Basin}

Streamflow gain with estimated amount in cubic feet per second-Table 7. The only gains or losses presented are those larger in magnitude than the uncertainty in computed streamflows

A U.S. Geological Survey streamflow-gaging station and map identifier-Table 1

on Spring

29 Reach number-Table 2

Figure 6. Streamflow gains and losses, March 15-21, 2010, lower Guadalupe River Basin, south-central Texas. 

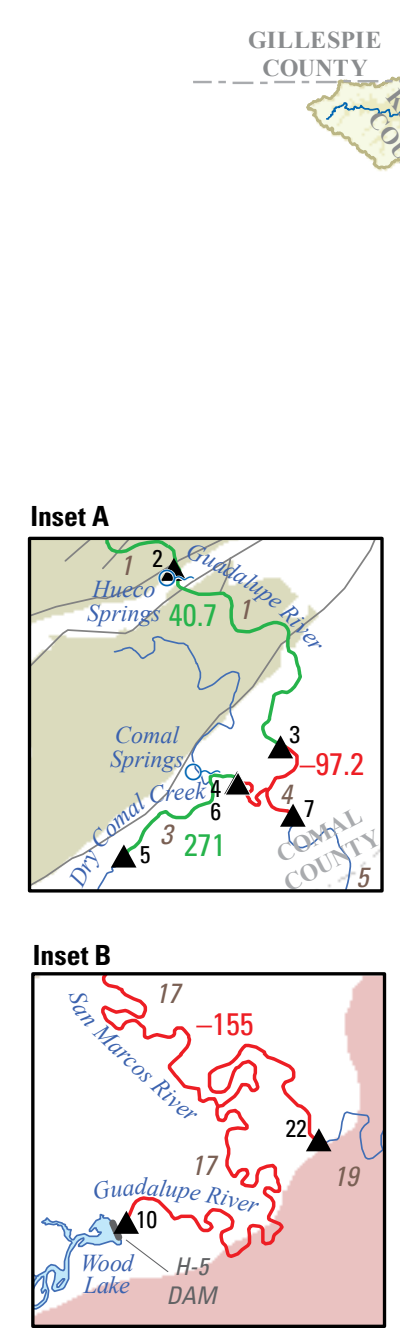

Base modified from U.S. Geological Survey 1:100,000 scale digital data

Universal Transverse Mercator, zone 14 North American Datum of 1983

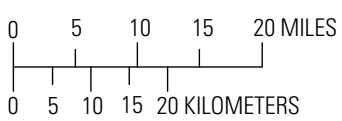

\section{Major aquifer system}

Gulf Coast

Carrizo-Wilcox

Edwards-Trinity

Edwards

Trinity

\section{Minor aquifer system}

Yegua Jackson

Sparta

Queen City

Fault

\section{Lower Guadalupe River Basin}

Streamflow gain or loss with estimated

amount in cubic feet per second-Table 8.

The only gains or losses presented are

those larger in magnitude than the

uncertainty in computed streamflows

\section{Streamflow gain}

Streamflow loss

$\Delta \quad$ U.S. Geological Survey streamflow-gaging station and map identifier-Table

\section{Spring}

29 Reach number-Table 2

\begin{tabular}{|c|c|c|}
\hline \multicolumn{3}{|c|}{$\begin{array}{l}\text { Mean daily streamflow measured at } \\
\text { selected sites during April 10-16, } 2011\end{array}$} \\
\hline $\begin{array}{c}\text { Map } \\
\text { identifier }\end{array}$ & $\begin{array}{l}\text { U.S. Geological } \\
\text { Survey station } \\
\text { number }\end{array}$ & $\begin{array}{c}\text { Streamflow, } \\
\text { in cubic feet } \\
\text { per second }\end{array}$ \\
\hline 8 & 08169 & \\
\hline 22 & 08173900 & 465 \\
\hline
\end{tabular}

Figure 7. Streamflow gains and losses, April 10-16, 2011, lower Guadalupe River Basin, south-central Texas. 


\section{Streamflow Gains and Losses during August 19-25, 2011}

The August 19-25, 2011, period represents base-flow conditions during the most severe drought conditions since the 1950s (Winters, 2013). During the August 19-25, 2011, base-flow period, three reaches had gains greater than the uncertainty in the computed streamflow (fig. 8; table 9). Reach 3 on the Comal River gained $168 \mathrm{ft}^{3} / \mathrm{s}$; nearly all of this gain represents inflow from Comal Springs as in the March 2010 and April 2011 base-flow periods. Reach 21 on Sandies Creek gained a small amount of flow $\left(0.092 \mathrm{ft}^{3} / \mathrm{s}\right)$. Reach 27 , which includes Coleto Creek, Perdido Creek, and Coleto Creek Reservoir, gained $1.98 \mathrm{ft}^{3} / \mathrm{s}$, likely as a result of groundwater inflows from the Gulf Coast aquifer, which are enhanced along this reach by seepage losses from Coleto Creek Reservoir. During this period of extreme drought, five reaches had losses greater in magnitude than the uncertainty in the computed streamflow (fig. 8). Reach 4, including the confluence of the Guadalupe and Comal Rivers, lost $82.8 \mathrm{ft}^{3} / \mathrm{s}$. Reach 5 on the Guadalupe River lost $53.5 \mathrm{ft}^{3} / \mathrm{s}$, with part of that loss possibly occurring as infiltration to the alluvium of the streambed or as recharge to the Carrizo-Wilcox aquifer outcrop (figs. 2-3). Reaches 9 and 10 of the Blanco River lost 2.20 and 6.60 $\mathrm{ft}^{3} / \mathrm{s}$, respectively, likely as infiltration through numerous faults (Hanson and Small, 1995; U.S. Geological Survey, $2012 b$ ) intersecting the stream channel northwest of Kyle (fig. 1). Whereas reach 15 on Plum Creek between Lockhart and Luling gained streamflow during the March 2010 and April 2011 assessments, reach 15 lost $2.11 \mathrm{ft}^{3} / \mathrm{s}$ during the August 2011 assessment. Water levels in the Carrizo-Wilcox aquifer were likely much lower in August 2011 compared to March 2010 and April 2011, causing the stream to lose water to the Carrizo-Wilcox aquifer or to alluvial terrace deposits of the Leona Formation that by August 2011 were dried out compared to the previous assessments.

\section{Streamflow Gains and Losses during September 22-28, 2012}

Analysis of the September 22-28, 2012, base-flow period was only done for reaches $6,7,16$, and 17 on the Guadalupe River between Seguin, Tex., and Gonzalez, Tex. Inflows from San Marcos River and Plum Creek (fig. 9) were included in this assessment. Because USGS streamflow-gaging station 08173500 San Marcos River at Ottine, Tex., was not active during 2012, the streamflows for USGS streamflow-gaging stations 08172000 San Marcos River at Luling, Tex., and 08173000 Plum Creek near Luling, Tex., were included in the assessment of the Guadalupe River reach between Seguin, Tex., and Gonzalez, Tex. During the September 22-28, 2012, base-flow period, no computed gains or losses were greater in magnitude than the uncertainty in the computed streamflow (table 10). 

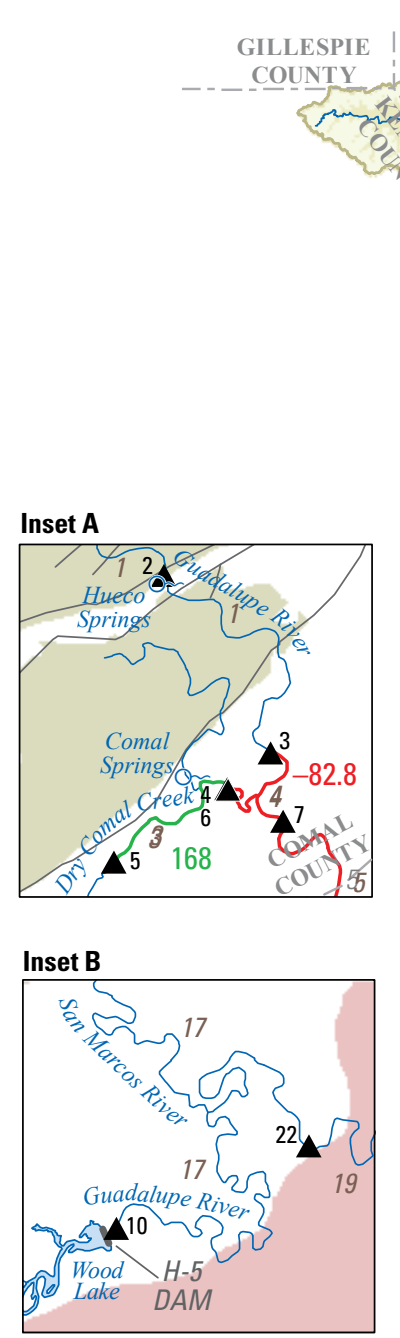

Base modified from U.S. Geological Survey 1:100,000 scale digital data Universal Transverse Mercator, zone 14 North American Datum of 1983

\section{EXPLANATION}

Major aquifer system

Gulf Coast

Carrizo-Wilcox

Edwards-Trinity

Edwards

Trinity

\section{Minor aquifer system}

Yegua Jackson

Sparta

Queen City

Fault

\section{Lwer Guadalupe River Basin}

Streamflow gain or loss with estimated amount in cubic feet per second-Table 9 .

The only gains or losses presented are

those larger in magnitude than the

uncertainty in computed streamflows

Streamflow gain

Streamflow loss

A.S. Geological Survey streamflow-gaging station and map identifier-Table 1

\section{Spring}

29 Reach number-Table 2

Figure 8. Streamflow gains and losses, August 19-25, 2011, lower Guadalupe River Basin, south-central Texas.

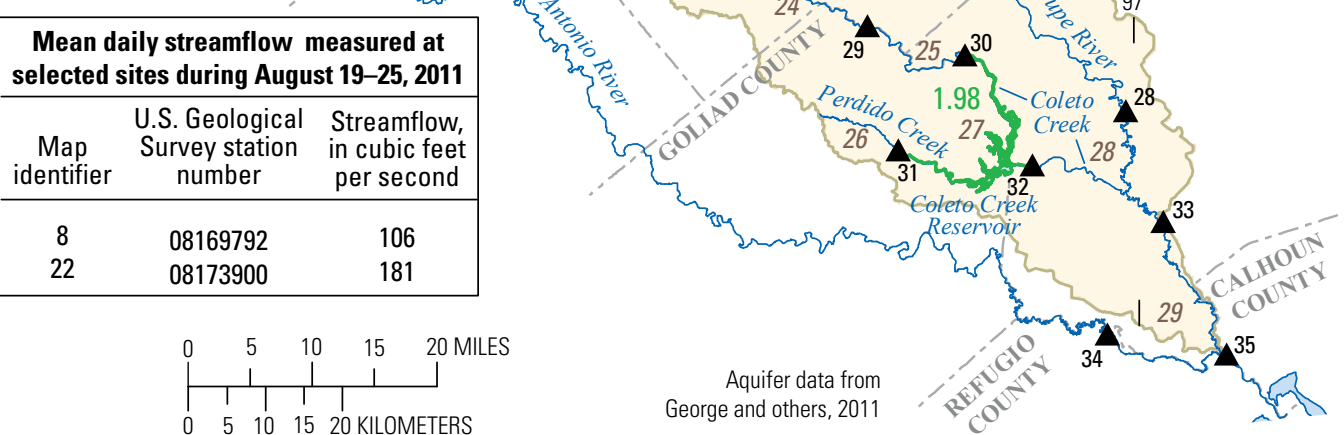



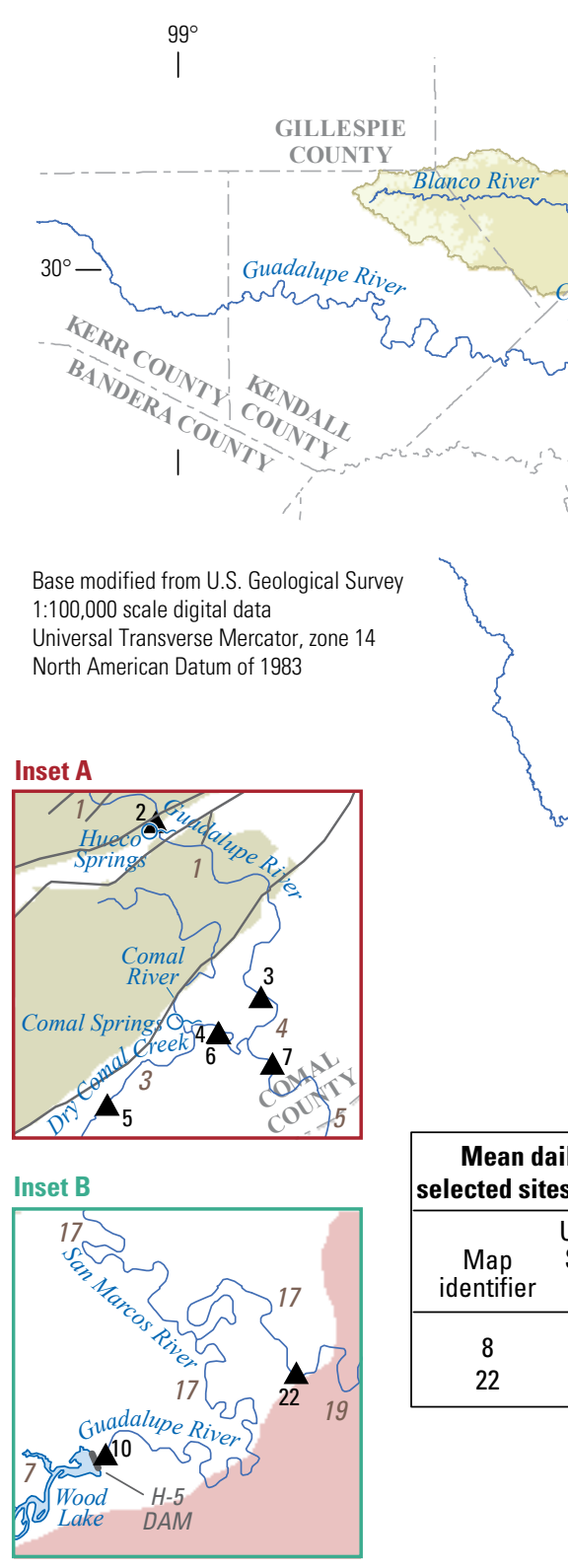

名i

$98^{\circ}$<smiles>C1#CC2CC1C2</smiles>

;

\section{Major aquifer system}

Gulf Coast

Carrizo-Wilcox

Edwards-Trinity

Edwards

Trinity

\section{Minor aquifer system}

Sparta

Queen City

- Fault

ower Guadalupe River Basin

- - Boundary for extent of gain-loss analysis for September 22-28, 2012

9 U.S. Geological Survey streamflow-gaging station and map identifier-Table

Or Spring

29 Reach number-Table 2

Note: No gains or losses were larger in magnitude than the uncertainty in computed streamflows during September 22-28, 2012 (table 10)

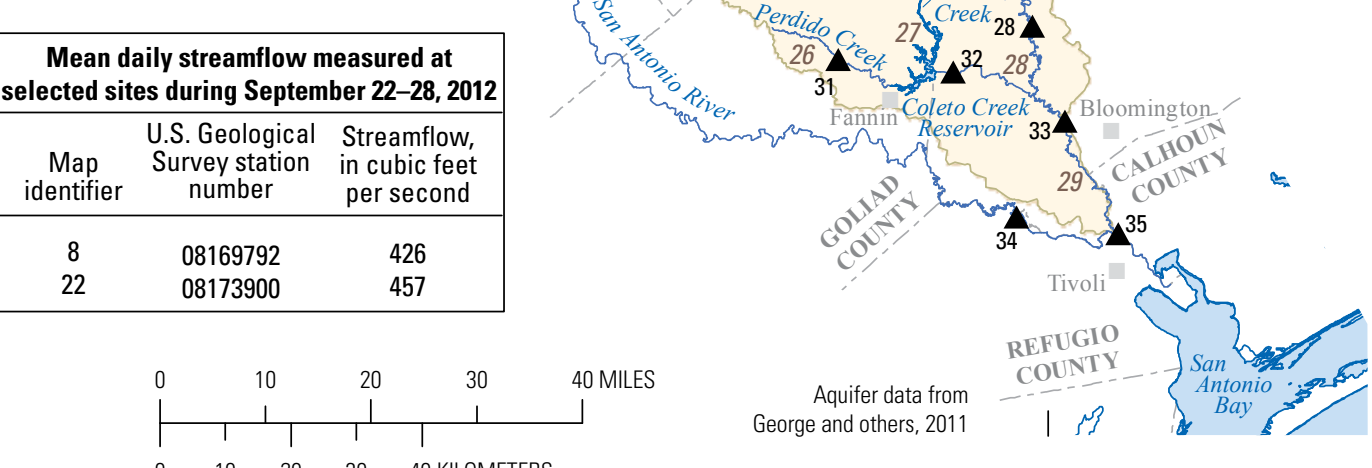

Figure 9. Streamflow gains and losses, September 22-28, 2012, in a reach of the Guadalupe River from Seguin, Texas, to Gonzales, Tex., lower Guadalupe River Basin, southcentral Texas. 


\section{Summary}

This report describes streamflow in the lower Guadalupe River Basin, consisting of the Guadalupe River drainage basin downstream from Canyon Lake to the Guadalupe River near Tivoli, Texas, during selected base-flow periods in 2010-12. The U.S. Geological Survey, in cooperation with the U.S. Army Corps of Engineers-Fort Worth District, the Texas Water Development Board, the Guadalupe-Blanco River Authority, and the Edwards Aquifer Authority, investigated streamflow gains and losses in the lower Guadalupe River Basin during four base-flow periods in March 2010, April 2011, August 2011, and September 2012. Streamflow and springflow data were collected at 35 streamflow-gaging stations (including 6 deployed for this study) in the study area during the selected base-flow periods from 2010-11. The assessment of streamflow in September 2012 was limited to the Guadalupe River between Seguin, Tex., and Gonzales, Tex., and the San Marcos River between Luling, Tex., and Gonzales, Tex.

During the March 15-21, 2010, base-flow period, five reaches had gains greater than the uncertainty in the computed streamflow. Reach 1 on the Guadalupe River downstream from Canyon Lake gained 130 cubic feet per second $\left(\mathrm{ft}^{3} / \mathrm{s}\right)$. This gain primarily is from groundwater inflow from the Edwards aquifer outcrop, which includes a large contribution from Hueco Springs. Reach 3 on the Comal River gained $359 \mathrm{ft}^{3} / \mathrm{s}$, nearly all of which represents the inflow from Comal Springs. Reach 15 on Plum Creek between Lockhart and Luling gained $19.8 \mathrm{ft}^{3} / \mathrm{s}$, which likely represents inflow from the CarrizoWilcox aquifer or from alluvial terrace deposits of the Leona Formation. Reach 21 on Sandies Creek gained $9.4 \mathrm{ft}^{3} / \mathrm{s}$, which might be related to inflows from the Yegua Jackson aquifer that is composed primarily of the Yegua Formation. Reach 27 gained $16.2 \mathrm{ft}^{3} / \mathrm{s}$, which likely is a result of groundwater inflows from the Gulf Coast aquifer that are enhanced by seepage losses from Coleto Creek Reservoir. During this period of elevated base flow in March 2010, streamflow losses greater than the uncertainty in the computed streamflow were not measured in any of the reaches.

During the April 10-16, 2011, base-flow period, three reaches had gains greater than the uncertainty in the computed streamflow. Reach 1 on the Guadalupe River gained 40.7 $\mathrm{ft}^{3} / \mathrm{s}$; this gain was likely caused by inflows from the Edwards aquifer and Hueco Springs. Reach 3 on the Comal River gained $271 \mathrm{ft}^{3} / \mathrm{s}$, nearly all of which represents the inflow from Comal Springs. Reach 15 on Plum Creek between Lockhart and Luling gained $3.24 \mathrm{ft}^{3} / \mathrm{s}$, which is likely related to inflows from the Carrizo aquifer or inflows from alluvial terrace deposits of the Leona Formation. During this base-flow period, losses greater in magnitude than the uncertainty in the computed streamflow were measured in three reaches. Reach 4 on the Guadalupe River near New Braunfels, Tex., lost 97.2 $\mathrm{ft}^{3} / \mathrm{s}$. Reach 10, the Blanco River near Kyle, lost $18.7 \mathrm{ft}^{3} / \mathrm{s}$, and reach 17, which includes the confluence of the Guadalupe and San Marcos Rivers, lost $155 \mathrm{ft}^{3} / \mathrm{s}$.
During the August 19-25, 2011, base-flow period, three reaches had gains greater than the uncertainty in the computed streamflow. Reach 3 on the Comal River gained $168 \mathrm{ft}^{3} / \mathrm{s}$, nearly all of which represents inflow from Comal Springs. Reach 21 on Sandies Creek gained $0.092 \mathrm{ft}^{3} / \mathrm{s}$. Reach 27, including Coleto Creek, Perdido Creek, and Coleto Creek Reservoir, gained $1.98 \mathrm{ft}^{3} / \mathrm{s}$, likely as a result of groundwater inflows from the Gulf Coast aquifer, which are enhanced in the area near reach 27 by seepage losses to the groundwater system from Coleto Creek Reservoir. During this period of extreme drought, five reaches had losses greater in magnitude than the uncertainty in the computed streamflow. Reach 4, including the confluence of the Guadalupe and Comal Rivers, lost $82.8 \mathrm{ft}^{3} / \mathrm{s}$. Reach 5 on the Guadalupe River lost 53.5 $\mathrm{ft}^{3} / \mathrm{s}$, with part of that loss possibly occurring as infiltration to the alluvium of the streambed or as recharge to the CarrizoWilcox aquifer outcrop. Reaches 9 and 10 of the Blanco River lost 2.20 and $6.60 \mathrm{ft}^{3} / \mathrm{s}$, respectively, likely through the numerous faults that intersect the stream channel northwest of Kyle. Reach 15 on Plum Creek between Lockhart and Luling lost $2.11 \mathrm{ft}^{3} / \mathrm{s}$; this loss was also likely in the form of recharge to the Carrizo-Wilcox aquifer outcrop.

Analysis of the September 22-28, 2012, base-flow period was limited to reaches $6,7,16$, and 17 on the Guadalupe River between Seguin, Tex., and Gonzalez, Tex., including flows from San Marcos River and Plum Creek. The streamflow-gaging station San Marcos River at Ottine, Tex., was not active during 2012 so the streamflows for San Marcos River at Luling, Tex., and Plum Creek near Luling, Tex., were included in the assessment of the Guadalupe River reach between Seguin, Tex., and Gonzalez, Tex. During the September 22-28, 2012, base-flow period no computed gains or losses were greater in magnitude than the uncertainty in the computed streamflow.

\section{References Cited}

Aronow, Saul., Brown, T.E., Brewton, J.L., Eargle, D.H., and Barnes, V.E., 1987, Geologic atlas of Texas, GA0005 Beeville-Bay City sheet: Austin, The University of Texas, Bureau of Economic Geology, 4 p., 1 sheet.

Brown, T.E., Waechter, N.B., Owens, Frith, Howeth, Ike, and Barnes, V.E., 1976, Geologic atlas of Texas, GA0011 Crystal-City Eagle Pass sheet: Austin, The University of Texas, Bureau of Economic Geology, 6 p., 1 sheet.

Brown, T.E., Waechter, N.B., and Barnes, V.E., 1983, Geologic atlas of Texas, GA0029 San Antonio sheet: Austin, The University of Texas, Bureau of Economic Geology, 8 p., 1 sheet.

Brune, G.M., 1975, Major and historical springs of Texas: Texas Water Development Board Report 189, 91 p.

Environmental Systems Research Institute, Inc., 2008, ArcMap, GIS software, Version 9.2: Redlands, Calif. 
Fulford, J.M., 1992, Characteristics of U.S. Geological Survey discharge measurements for water year 1990: U.S. Geological Survey Open-File Report 92-493, 79 p.

George, P.G., Mace, R.E., and Petrossian, Rima, 2011, Aquifers of Texas: Texas Water Development Board Report 380,172 p.

Granato, G.E., 2009, Computer programs for obtaining and analyzing daily mean streamflow data from the U.S. Geological Survey National Water Information System Web Site: U.S. Geological Survey Open-File Report 2008-1362, $123 \mathrm{p}$.

Guadalupe-Blanco River Authority, 2007a, Canyon Lake fact sheet: accessed October 10, 2007, at http://www.gbra.org/ Public/CanyonReservoir.aspx.

Guadalupe-Blanco River Authority, 2007b, Coleto Creek Park and reservoir fact sheet: Guadalupe-Blanco River Authority, accessed October 10, 2007, at http://www.gbra. org/Recreation/ColetoCreek/Default.aspx.

Hanson, J.A., and Small, T.A., 1995, Geologic framework and hydrogeologic characteristics of the Edwards aquifer outcrop, Hays County, Texas: U.S. Geological Survey Water-Resources Investigations Report: 95-4265, 10 p.

Helsel, D.R., and Hirsch, R.M., 2002, Statistical methods in water resources-Hydrologic analysis and interpretation: U.S. Geological Survey Techniques of Water-Resources Investigations, book 4, chap. A3, 510 p., accessed March 2008, at http://pubs.usgs.gov/twri/twri4a3/.

Hirsch, R.M., 1982, A comparison of four streamflow record extension techniques: Water Resources Research, v. 18, no. 4 , p. 1081-1088.

Larkin, T.J., and Bomar, G.W., 1983, Climatic atlas of Texas: Texas Department of Water Resources Limited Printing Report LP-192, 151 p.

Lizárraga, J.S., and Ockerman, D.J., 2010, Simulation of streamflow, evapotranspiration, and groundwater recharge in the lower San Antonio River watershed, south-central Texas, 2000-2007: U.S. Geological Survey Scientific Investigations Report 2010-5027, 41 p.

Multi-Resolution Land Characteristics Consortium, 2006, National Land Cover Database 2006: U.S. Geological Survey, accessed June 10, 2013, at http://www.mrlc.gov/ nlcd06_data.php.

National Conference of State Legislatures, 2013, State Water Law Regulations - Texas: accessed August 20, 2013, at http://www.ncsl.org/issues-research/env-res/state-waterwithdrawal-regulations.aspx\#tx.
National Oceanic and Atmospheric Administration, 2002, Climatography of the United States 81, Monthly station normals of temperature, precipitation, and heating and cooling degree days 1971-2000, Texas: Asheville, N.C., National Climatic Data Center, v. 41, 88 p.

National Oceanic and Atmospheric Administration, 2012, Preliminary monthly climate data (CF6), New Braunfels, Texas, August 2011 \& September 2011 (Archived Data): National Weather Service, accessed August 15, 2012, at http://www.nws.noaa.gov/climate/index.php?wfo=ewx.

National Oceanic and Atmospheric Administration, 2013, Advanced hydrologic prediction service, accessed February 22, 2013, at http://water.weather.gov/precip/.

Novak, C.E., 1985, WRD data reports preparation guide: U.S. Geological Survey Open File Report 85-480, 333 p.

Ockerman, D.J., and Slattery, R.N., 2008, Streamflow conditions in the Guadalupe River Basin, south-central Texas, water years 1987-2006-An assessment of streamflow gains and losses and relative contribution of major springs to streamflow: U.S. Geological Survey Scientific Investigations Report 2008-5165, 22 p.

Oklahoma State University Department of Chemistry, 2001, Vapor pressure of water: Oklahoma State University Department of Chemistry, accessed on September 12, 2012, at http://intro.chem.okstate.edu/1515sp01/database/vpwater. html.

Ott, R.L., 1988, An introduction to statistical methods and data analysis ( $3 \mathrm{~d}$ ed.): Boston, Mass., PWS-Kent Publishing Company, $945 \mathrm{p}$.

Pantea, M.P., and Cole, J.C., 2004, Three-dimensional geologic framework modeling of faulted hydrostratigraphic units within the Edwards aquifer, northern Bexar County, Texas: U.S. Geological Survey Scientific Investigations Report 2004-5226, 10 p.

Penman, H.L., 1948, Natural evaporation from open water, bare soil and grass: Proceedings of the Royal Society of London, Series A, Mathematical and Physical Sciences, v. 193, no. 1032, p. 120-45.

Proctor, C.V., Jr., Brown, T.E., Waechter, N.B., Aronow, Saul, and Barnes, V.E., 1974, Geologic atlas of Texas, GA0030 Seguin sheet: Austin, The University of Texas, Bureau of Economic Geology, 6 p., 1 sheet.

Puente, Celso, 1978, Method of estimating natural recharge to the Edwards aquifer in the San Antonio area, Texas: U.S. Geological Survey Water-Resources Investigations Report: 78-10, 34 p. 
Slade, R.M., Jr., Dorsey, M.E., and Stewart, S.L., 1986, Hydrology and water quality of the Edwards aquifer associated with Barton Springs in the Austin area, Texas: U.S. Geological Survey Water-Resources Investigations Report 86-4036, 117 p.

Texas Commission on Environmental Quality, 2011, Water rights database and related files: Texas Commission on Environmental Quality, accessed July 23, 2011, at http://www.tceq.texas.gov/permitting/water_rights/wr_ databases.html.

Texas Water Development Board, 2013, Precipitation \& lake evaporation: Texas Water Development Board, accessed January 30, 2013, at https://www.twdb.state.tx.us/ surfacewater/conditions/evaporation/index.asp.

Turco, M.J., East, J.W., and Milburn, M.S., 2007, Base flow (1966-2005) and streamflow gain and loss (2006) of the Brazos River, McLennan County to Fort Bend County, Texas: U.S. Geological Survey Scientific Investigations Report 2007-5286, 27 p.

Turnipseed, D.P., and Sauer, V.B., 2010, Discharge measurements at gaging stations: U.S. Geological Survey Techniques and Methods book 3, chap. A8, 87 p. (Also available at http://pubs.usgs.gov/tm/tm3-a $8 /$. )

University of Nebraska-Lincoln, 2013, The drought monitor: National Drought Mitigation Center, accessed August 2013, at http://droughtmonitor.unl.edu/.

U.S. Census Bureau, 2011, State \&county quick factsTexas: U.S. Census Bureau, accessed March 14, 2011, at http://quickfacts.census.gov/qfd/states/48000.html.
U.S. Environmental Protection Agency, 2004, NPDES reporting requirements handbook: Dallas, Tex, U.S. Environmental Protection Agency Region 6, 54 p.

U.S. Environmental Protection Agency, 2013, Enforcement \& compliance history online (ECHO): U.S. Environmental Protection Agency, accessed May 6, 2013, at http://www. epa-echo.gov/echo/compliance_report_water.html.

U.S. Geological Survey, 2012a, USGS water data for Texas: National Water Information System-Web Interface, accessed March 14, 2012, at http://waterdata.usgs.gov/tx/ nwis/nwis.

U.S. Geological Survey, 2012b, Texas geology: Mineral Resources On-Line Spatial Data, accessed September 12, 2012, at http://mrdata.usgs.gov/sgmc/tx.html.

U.S. Geological Survey, 2012c, Documentation: USGS Annual Water Data Report, accessed March 20, 2012, at http://wdr.water.usgs.gov/current/documentation.html.

Winters, K.E., 2013, A historical perspective on precipitation, drought severity, and streamflow in Texas during 1951-56 and 2011: U.S. Geological Survey Scientific Investigations Report 2013-5113, 24 p.

Wurbs, R.A., Sanchez-Torres, Gerardo, and Dunn, D.D., 1994, Reservoir/river system reliability considering water rights and water quality: Technical Report No. 165, Texas Water Resources Institute, Texas A\&M University, $193 \mathrm{p}$.
Publishing support provided by

Lafayette Publishing Service Center
Information regarding water resources in Texas is available at

http://tx.usgs.gov/ 
